MATHEMATICS OF COMPUTATION

Volume 72 , Number 242 , Pages $577-606$

S $0025-5718(02) 01464-3$

Article electronically published on November 20, 2002

\title{
ENHANCED ACCURACY BY POST-PROCESSING FOR FINITE ELEMENT METHODS FOR HYPERBOLIC EQUATIONS
}

\author{
BERNARDO COCKBURN, MITCHELL LUSKIN, CHI-WANG SHU, AND ENDRE SÜLI
}

\begin{abstract}
We consider the enhancement of accuracy, by means of a simple post-processing technique, for finite element approximations to transient hyperbolic equations. The post-processing is a convolution with a kernel whose support has measure of order one in the case of arbitrary unstructured meshes; if the mesh is locally translation invariant, the support of the kernel is a cube whose edges are of size of the order of $\Delta x$ only. For example, when polynomials of degree $k$ are used in the discontinuous Galerkin (DG) method, and the exact solution is globally smooth, the DG method is of order $k+1 / 2$ in the $L^{2}$-norm, whereas the post-processed approximation is of order $2 k+1$; if the exact solution is in $L^{2}$ only, in which case no order of convergence is available for the DG method, the post-processed approximation converges with order $k+1 / 2$ in $L^{2}\left(\Omega_{0}\right)$, where $\Omega_{0}$ is a subdomain over which the exact solution is smooth. Numerical results displaying the sharpness of the estimates are presented.
\end{abstract}

\section{INTRODUCTION}

In this paper, we consider general finite element methods for time-dependent linear hyperbolic systems of the form

$$
\begin{array}{ll}
u_{t}+\sum_{j=1}^{d} A_{j} u_{x_{j}}+A_{0} u=0, & (x, t) \in \mathbb{R}^{d} \times(0, T], \\
u(x, 0)=u_{0}(x), & x \in \mathbb{R}^{d},
\end{array}
$$

Received by the editor November 14, 2000.

2000 Mathematics Subject Classification. Primary 65M60, 65N30, 35L65.

Key words and phrases. Post-processing, finite element methods, hyperbolic problems.

The first author was supported in part by NSF Grant DMS-9807491 and by the University of Minnesota Supercomputing Institute.

The second author was supported in part by NSF Grant DMS 95-05077, by AFOSR Grant F49620-98-1-0433, by ARO Grant DAAG55-98-1-0335, by the Institute for Mathematics and its Applications, and by the Minnesota Supercomputing Institute.

The third author was supported in part by ARO Grant DAAG55-97-1-0318 and DAAD19-001-0405, NSF Grant DMS-9804985, NASA Langley Grant NCC1-01035 and and Contract NAS197046 while this author was in residence at ICASE, NASA Langley Research Center, and by AFOSR Grant F49620-99-1-0077.

The fourth author is grateful to the Institute for Mathematics and Its Applications at the University of Minnesota and the University of Minnesota Supercomputing Institute for their generous support. 
where $\left\{A_{j}\right\}_{j=1}^{d}$ are real, constant coefficient $m \times m$ matrices such that $\sum_{j=1}^{d} A_{j} \xi_{j}$ has real eigenvalues and a complete set of linearly independent eigenvectors for all $\xi \in \mathbb{R}^{d}$, and the function $u$ has range in $\mathbb{R}^{m}$. Our aim in this paper is to show how to exploit the inherently oscillatory nature of numerical solutions to this problem computed by means of finite element methods to enhance the quality of the approximation. This enhancement is achieved by post-processing the approximate solution only once, at the very end of the computation, at $t=T$. The post-processing considered here is completely independent of the partial differential equation under consideration and can be performed for entirely arbitrary triangulations; however, it takes a particularly simple and computationally efficient form when the triangulation is locally translation invariant.

To illustrate the basic idea, let us consider the following simple model problem:

$$
u_{t}+u_{x}=0, \quad \text { in }(0,1) \times(0, T), \quad u(x, 0)=\sin (2 \pi x) \quad \text { for } x \in(0,1),
$$

subject to periodic boundary conditions, and let us compute an approximation $U$ to its solution $u$ by using the discontinuous Galerkin (DG) method with piecewise polynomials of degree one over uniform grids of spacing $h$. We also consider the post-processed approximation $U^{\star}=K_{h}^{4,2} \star U$, where the convolution kernel $K_{h}^{4,2}(x)=\frac{1}{h} K^{4,2}(x / h)$ is defined by

$$
K^{4,2}(y)=-\frac{1}{12} \psi^{(2)}(y-1)+\frac{7}{6} \psi^{(2)}(y)-\frac{1}{12} \psi^{(2)}(y+1),
$$

where $\psi^{(2)}$ is the B-spline obtained by convolving the characteristic function $\psi^{(1)}=$ $\chi$ of the interval $(-1 / 2,1 / 2)$ with itself once. In Figure 1.1 we display, for $T=$ 0.1 and $h=1 / 10$ and $h=1 / 20$, the errors $x \mapsto u(T, x)-U(T, x)$ and $x \mapsto$ $u(T, x)-U^{\star}(T, x)$. The time-step was chosen so small that the overall accuracy of the method is dominated by the spatial error. We note the oscillatory nature of the error $x \mapsto u(T, x)-U(T, x)$ typical of finite element methods and the apparent superconvergence of the numerical solution at the two Gauss-Radau points, a fact discovered in 1995 by Adjerid, Aiffa, and Flaherty [2]; see also their recent work [1]. In contrast with this behavior, we observe the complete absence of oscillations from the error $u(T)-U^{\star}(T)$. This shows that convolving the approximate solution $U$ with the kernel $K_{h}^{4,2}$ filters out the numerical oscillations around the exact solution. Moreover, the result of such a filtering is a new approximation $U^{\star}$ that converges faster to $u$ than $U$. Indeed, in Figure 1.2, we display the functions $x \mapsto \log (|u(T, x)-U(T, x)|)$, for $h=1 / 10,1 / 20,1 / 40$ and $1 / 80$; we observe that each time $h$ is halved, the maximum of $x \mapsto\left|u(T, x)-U^{\star}(T, x)\right|$ is divided by a factor not less than eight. This indicates that the post-processed approximation is at least third-order convergent; the original approximate solution $U$ exhibits only second-order convergence.

In Figures 1.3 and 1.4 we repeat the above experiment using polynomials of degree two. Again we observe the oscillatory nature of the approximation and the superconvergence at the three Gauss-Radau points in Figure 1.3 (top), and that the oscillations are filtered out upon convolution in Figure1.3 (bottom). This time, the convolution kernel $K_{h}^{6,3}(x)=\frac{1}{h} K^{6,3}(x / h)$ is defined by

$$
\begin{gathered}
K^{6,3}(y)=\frac{37}{1920} \psi^{(4)}(y-2)-\frac{97}{480} \psi^{(4)}(y-1)-\frac{437}{320} \psi^{(4)}(y) \\
-\frac{97}{480} \psi^{(4)}(y+1)+\frac{37}{1920} \psi^{(4)}(y+2),
\end{gathered}
$$



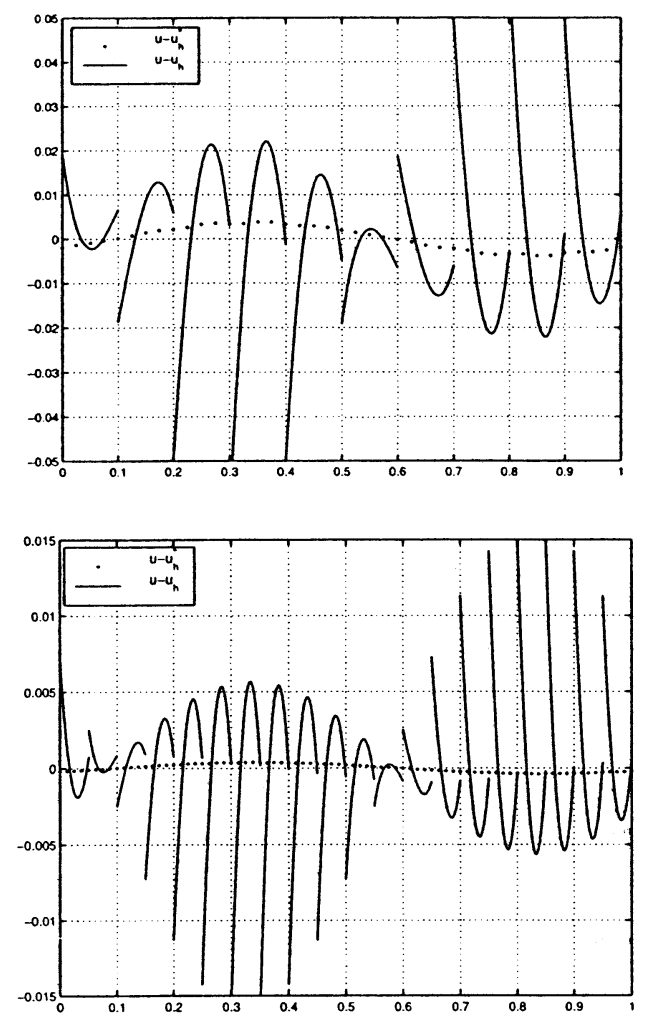

Figure 1.1. The errors $u-U$ (solid line) and $u-U^{\star}$ (dots) at $T=0.1$ for $h=1 / 10$ (top) and $h=1 / 20$ (bottom). The function $u$ is the smooth exact solution, $U$ is the approximation given by the DG method with polynomials of degree one, and $U^{\star}=K_{h}^{4,2} \star U$.

where $\psi^{(4)}$ is the B-spline obtained by convolving the characteristic function $\psi^{(1)}=$ $\chi$ of the interval $(-1 / 2,1 / 2)$ with itself three times. In Figure 1.4, we see that each time $h$ is halved, the maximum error decreases by a factor not less than thirty two. This shows that the error in the post-processed approximation is of fifth order.

In connection with this fact, we note here that in 1996 Lowrie [18] found analytical and numerical evidence that when polynomials of degree $k$ are used, a "component of the error" of the DG method converges with order $2 k+1$ in the $L^{2}$ norm. This fact stands in striking contrast with convergence of order $k+1 / 2$ for the underlying DG approximation $(k+1$ for the one-dimensional case and special grids in several space dimensions). In this paper, we provide a firm mathematical basis for this observation, and show how to compute the superconvergent approximation $U^{\star}$ by a simple post-processing technique which is independent of the equation and of the numerical method.

The paper is organized as follows. In Section 2 we present a brief account of the development of the ideas behind this paper. In Section 3 we state and discuss our main theoretical results, and in Section 4 we present their proofs. In Section 5 we display numerical experiments which not only verify our theoretical results but also 


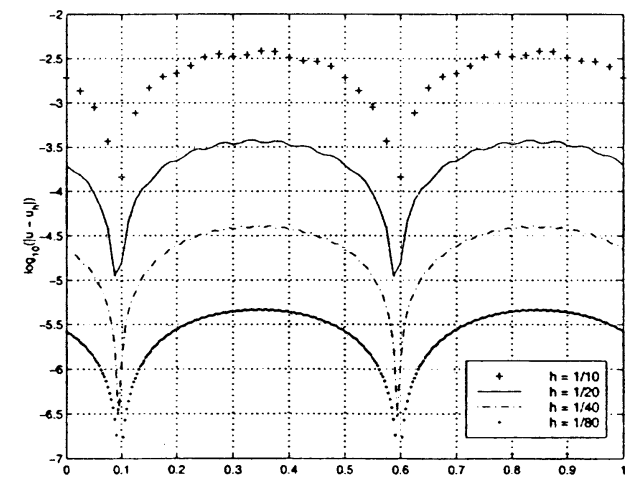

Figure 1.2. The errors $\log \left(\left|u-U^{\star}\right|\right)$ at $T=0.1$ for $h=1 / 10$ (top), $h=1 / 20, h=1 / 40$, and $h=1 / 80$ (bottom). Each time $h$ is halved, the maximum error decreases by a factor not less than 8 ; the order of convergence is, therefore, not less than 3 .
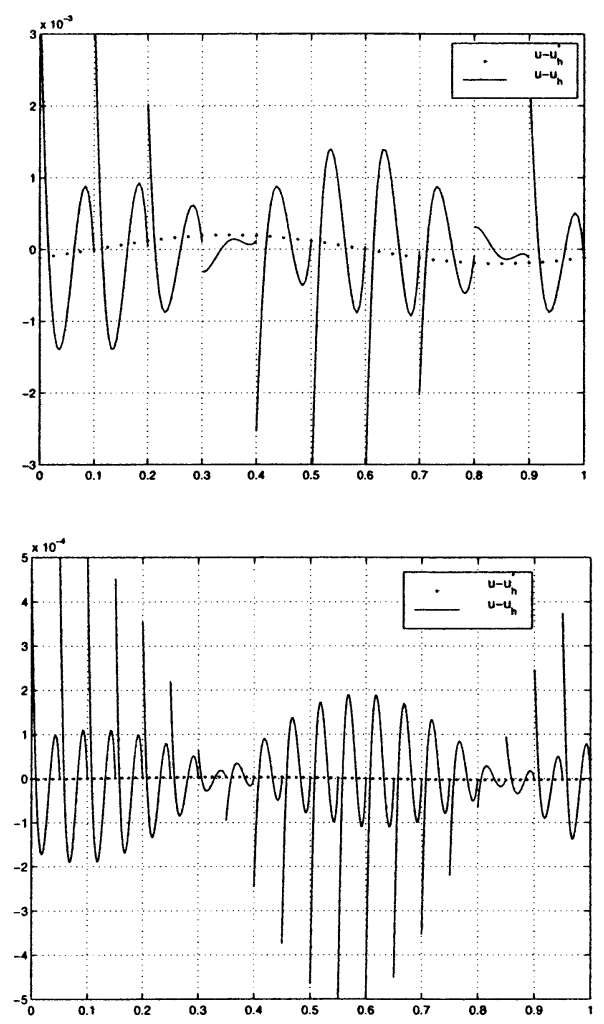

Figure 1.3. The errors $u-U$ (solid line) and $u-U^{\star}$ (dots) at $T=0.1$ for $h=1 / 10$ (top) and $h=1 / 20$ (bottom). The function $u$ is the smooth exact solution, $U$ is the approximation given by the DG method with polynomials of degree two, and $U^{\star}=K_{h}^{6,3} \star U$. 


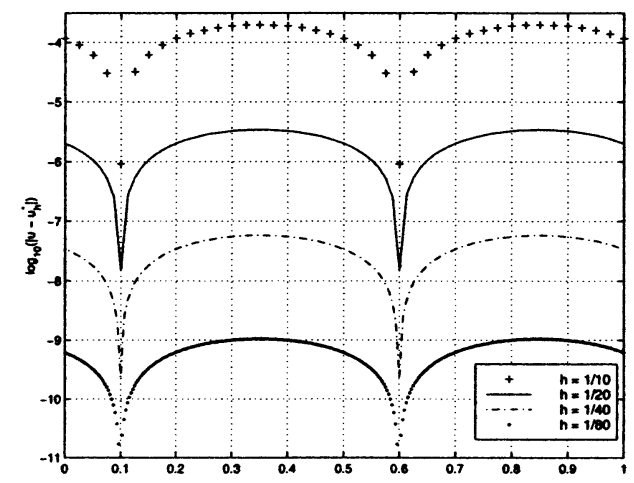

Figure 1.4. The errors $\log \left(\left|u-U^{\star}\right|\right)$ at $T=0.1$ for $h=1 / 10$ (top), $h=1 / 20, h=1 / 40$, and $h=1 / 80$ (bottom). Each time $h$ is halved, the maximum error decreases by a factor not less than 32 ; the order of convergence is, therefore, not less than 5 .

indicate how this kind of post-processing can be applied to convection-diffusion and nonlinear problems. We conclude, in Section 6, with some remarks.

\section{A BRIEF OVERVIEW OF THE DEVELOPMENT OF POST-PROCESSING TECHNIQUES}

In order to introduce the basic ideas of our work and to put them into proper perspective, we briefly review the development of post-processing techniques devised to improve the quality of numerical approximations. For further detail the reader should consult the monograph of Wahlbin 24] on superconvergence in Galerkin finite element methods.

2.1. Finite difference and spectral methods for hyperbolic problems. In 1977, Majda and Osher 20 considered formally high-order accurate dissipative difference schemes for hyperbolic problems. They studied a one-dimensional model problem of a two-by-two hyperbolic system whose characteristics are parallel to $x= \pm t$; the initial condition is a step function whose discontinuity is located at the origin. Majda and Osher showed that the rate of convergence on the region between the characteristics issuing from the origin, $|x / t|<1-\delta^{2}$, is independent of the numerical scheme. They pointed out that in 1962 Fedorenko [12] and in 1969 Apelkrans 3 displayed numerical evidence that the order of convergence had to be one. However, by selecting a suitable approximation of the initial datum, Majda and Osher showed that the order of convergence can be increased to two. Moreover, they found that they could recover the full formal order of accuracy of the scheme on the region $|x / t|<1-\delta^{2}$ provided they preprocessed the initial data in an appropriate way. In 1986, Johnson and Pitkäranta [16] used a similar idea in the analysis of the DG method for linear hyperbolic problems. The question of post-processing the initial data is considered in the book of Brenner, Thomée and Wahlbin [6]; see also the work of Jovanović, Ivanović and Süli [17] concerning the use of convolution mollifiers with B-spline kernels for second-order hyperbolic boundary value problems with nonsmooth data. 
In 1978, Mock and Lax [21] showed that for a difference scheme of any formal order of accuracy $\mu$, for linear hyperbolic systems, the moments of the exact solution converge with order $\mu$ provided that, again, the initial data was suitably preprocessed. This result holds even if the exact solution contains discontinuities. They also showed how to post-process the approximate solution by a simple convolution to enhance its accuracy over regions of smoothness of the exact solution: if the solution was sufficiently smooth locally, they could obtain nearly the full order of convergence $\mu$ provided that the support of the kernel was of order almost one. This seems to have been the first instance when the ideas of (i) preprocessing the initial data, (ii) obtaining error estimates for the moments, and (iii) post-processing the approximation, appear clearly delineated.

Later, in 1985, Gottlieb and Tadmor [13, motivated by the work of Mock and Lax [21, found a spectrally accurate post-processing kernel for spectral methods; see also the 1978 paper by Majda, McDonough and Osher [19. Again, the full spectral accuracy could be recovered by using a convolution; the measure of the support of the kernel had to be of order one.

2.2. Finite element methods for elliptic and parabolic problems. Quite independently of the developments reviewed above, in 1977 Bramble and Schatz 5] considered linear elliptic problems and showed how to post-process the finite element solution by means of a simple convolution to enhance the quality of the approximation. They showed that the order of convergence could be doubled if the exact solution was locally smooth. In 1977, Thomée 22] extended the work of Bramble and Schatz [5] to include superconvergence of the derivatives and gave an elegant proof of their approximation results by using Fourier analysis. In 1980, he also extended these results [23] to semidiscrete Galerkin finite element methods for parabolic problems.

It is important to point out that, just like Mock and Lax, Bramble and Schatz proved a negative-order norm error estimate (an error estimate of the moments in Mock and Lax's terminology) and then showed how to use it to enhance the approximation by a convolution. However, unlike Mock and Lax's convolution kernel, for locally translation invariant grids the Bramble-Schatz kernel has support in a cube whose diameter is of order $h$ only; this fact represents a considerable advantage from the computational point of view.

In 1981, Johnson and Nävert [15] applied this technique to steady-state advection-diffusion problems with small diffusion; they considered the standard Galerkin and the streamline-diffusion methods. An application of this technique to the simulation of miscible displacement was devised and analyzed in 1985 by Douglas [10. Other applications can be found in the book of Wahlbin [24.

2.3. The main ideas. It seems that the first (and only) attempt to apply this technique to finite element methods for hyperbolic problems was carried out in 1993 by Bales [4] who considered a fourth-order accurate finite element method applied to the one-dimensional wave equation. In this paper, we apply in a systematic way the ideas of Mock and Lax [21] and Bramble and Schatz [5] to enhance the accuracy of finite element approximations to hyperbolic problems by post-processing.

We proceed as follows. First, we obtain an estimate of the error between the analytical solution $u$ and the post-processed numerical approximation $U$ in terms of negative-order Sobolev norms of $u-U$. This result does not depend on the 
partial differential equation under consideration or on the numerical scheme. Next, we obtain negative-order norm a priori estimates for the error between the exact solution of a hyperbolic problem and its finite element approximation $U$. The final error estimate is then obtained by combining the above bounds.

\section{The RESUlts}

In this section, we present and discuss our main theoretical results.

3.1. An approximation result. We begin by presenting a result that relates negative-order norm a priori estimates of the difference between $u$ and an arbitrary approximation $U$ for $u$ to $L^{2}$-error estimates of the difference between $u$ and the post-processed counterpart $U$.

Let us recall the definition of a negative-order Sobolev norm on an open set $\Omega \subset \mathbb{R}^{d}$. We denote by $\|u\|_{0, \Omega}$ the standard $L^{2}$-norm of $u$ on $\Omega$. For any natural number $\ell$, we consider the norm and seminorm of the Sobolev space $H^{\ell}(\Omega)$, defined by

$$
\|u\|_{\ell, \Omega}=\left\{\sum_{|\alpha| \leq \ell}\left\|D^{\alpha} u\right\|_{0, \Omega}^{2}\right\}^{1 / 2}, \quad|u|_{\ell, \Omega}=\left\{\sum_{|\alpha|=\ell}\left\|D^{\alpha} u\right\|_{0, \Omega}^{2}\right\}^{1 / 2} .
$$

Sobolev norms and seminorms for vector-valued functions from $H^{\ell}\left(\Omega, \mathbb{R}^{m}\right)$ are defined analogously and are denoted by the same symbol as in the scalar case. We then define the negative order Sobolev norm $\|\cdot\|_{-\ell, \Omega}, \ell \geq 1$, by

$$
\|u\|_{-\ell, \Omega}=\sup _{\phi \in \mathcal{C}_{0}^{\infty}(\Omega)} \frac{\int_{\Omega} u(x) \phi(x) \mathrm{d} x}{\|\phi\|_{\ell, \Omega}} .
$$

Negative-order norms can be used to detect the oscillations of a function around zero. For example, for $\Omega=[-1,1], \ell \geq 1$ and $u_{N}(x)=\sin (2 \pi N x)$, a simple computation gives $\left\|u_{N}\right\|_{-\ell, \Omega}=1 /(2 \pi N)^{\ell}$, indicating that $u_{N}$ oscillates about zero in a very regular manner.

Next, we describe the type of post-processing to be considered following Bramble and Schatz [5]. We post-process the approximate solution by convolving it with a kernel $K_{H}^{\nu, \ell}(x)=K^{\nu, \ell}(x / H) / H^{d}$ which has to satisfy three properties. The first of these is that $K^{\nu, \ell}$ has compact support. The second is that it reproduces polynomials $p$ of degree $\nu-1$ by convolution, that is,

$$
K^{\nu, \ell} \star p=p
$$

This is the type of kernel used by Mock and Lax [21. The kernels used by Bramble and Schatz [5], which we shall next describe, have the further property that they are linear combinations of B-splines. Let $\chi$ be the characteristic function of the interval $(-1 / 2,1 / 2)$ and let $\delta$ denote the Dirac distribution concentrated at $x=0$. Then, we define recursively the functions $\psi^{(i)}$ as

$$
\psi^{(0)}=\delta, \quad \psi^{(n+1)}=\psi^{(n)} \star \chi, \text { for } n \geq 0,
$$

and, given an arbitrary multi-index $\alpha=\left(\alpha_{1}, \ldots, \alpha_{d}\right)$ and $y=\left(y_{1}, \ldots, y_{d}\right) \in \mathbb{R}^{d}$, we set

$$
\psi^{(\alpha)}(y)=\psi^{\left(\alpha_{1}\right)}\left(y_{1}\right) \cdots \psi^{\left(\alpha_{d}\right)}\left(y_{d}\right)
$$


We also set $\mathbf{1}=(1, \ldots, 1)$. The third and final property of the kernels considered here is that they are of the form

$$
K^{\nu, \ell \mathbf{1}}(y)=\sum_{\gamma \in \mathbb{Z}^{d}} k_{\gamma}^{\nu, \ell \mathbf{1}} \psi^{(\ell \mathbf{1})}(y-\gamma)
$$

where $k_{\gamma}^{\nu, \ell 1} \in \mathbb{R}$. Note that since the support of $K^{\nu, \ell \mathbf{1}}$ has been assumed compact, there are only finitely many nonzero coefficients $k_{\gamma}^{\nu, \ell 1}$ in this sum.

The imposition of these hypotheses is motivated by the following observations: the compactness of the support of the convolution kernel is advantageous from the computational point of view; the second property ensures that the accuracy of order $\nu$ is not destroyed by post-processing; the third property allows us to express derivatives of the convolution with the kernel in terms of simple difference quotients. Indeed, it is very easy to verify that for multi-indices $\alpha$ and $\beta$ such that $\beta_{i} \geq \alpha_{i}$ for $i=1, \ldots, d$, we have

$$
D^{\alpha}\left(\psi_{H}^{(\beta)} \star v\right)=\psi_{H}^{(\beta-\alpha)} \star \partial_{H}^{\alpha} v,
$$

where $\psi_{H}^{(\alpha)}(x)=\psi^{(\alpha / H)} / H^{d}$,

$$
\partial_{H}^{\alpha}:=\partial_{H, 1}^{\alpha_{1}} \cdots \partial_{H, d}^{\alpha_{d}} \quad \text { and } \quad \partial_{H, j} v(x)=\frac{1}{H}\left(v\left(x+\frac{1}{2} H e_{j}\right)-v\left(x-\frac{1}{2} H e_{j}\right)\right) .
$$

This fact can then be exploited in the finite element framework, as will be seen later. We are now ready to state an approximation result which shows that local smoothness of $u$ on the one hand and negative-order norm estimates of divided differences of the error $u-U$ on the other lead to a local bound on $u-K_{H}^{\nu, \ell 1} \star U$ in the $L^{2}$-norm.

Theorem 3.1 (Bramble and Schatz [5]). Let $\nu$ and $\ell$ be two natural numbers. Suppose, further, that $K_{H}^{\nu, \ell \mathbf{1}}(x)=K^{\nu, \ell \mathbf{1}}(x / H) / H^{d}$ where $K^{\nu, \ell \mathbf{1}}$ is a function of compact support which reproduces polynomials of degree $\nu-1$ by convolution, and which is the linear combination of B-splines, as in (3.1). Let $U$ be a function in $L^{2}\left(\Omega_{1}\right)$, where $\Omega_{1}$ is an open set in $\mathbb{R}^{d}$, and let $u$ be a function in $H^{\nu}\left(\Omega_{1}\right)$. Let $\Omega_{0}$ be an open set in $\mathbb{R}^{d}$ such that $\Omega_{0}+2 \operatorname{supp}\left(K_{H}^{\nu, \ell \mathbf{1}}\right) \Subset \Omega_{1}$ for all $H \leq H_{0}$. Then, for $H \leq H_{0}$, we have

$$
\left\|u-K_{H}^{\nu, \ell 1} \star U\right\|_{0, \Omega_{0}} \leq \frac{H^{\nu}}{\nu !} C_{1}|u|_{\nu, \Omega_{1}}+C_{1} C_{2} \sum_{|\alpha| \leq \ell}\left\|\partial_{H}^{\alpha}(u-U)\right\|_{-\ell, \Omega_{1}},
$$

where $C_{1}=\sum_{\gamma \in \mathbb{Z}^{d}}\left|k_{\gamma}^{\nu, \ell \mathbf{1}}\right|$ and $C_{2}$ depends solely on $\Omega_{0}, \Omega_{1}, d, \nu$, and $\ell$.

To illustrate the importance of this result, let us assume that there exist real numbers $\mu \geq 0$ and $a \in[0, \ell]$ such that, for all $H \leq H_{0}$,

$$
\sum_{|\alpha| \leq \ell}\left\|\partial_{H}^{\alpha}(u-U)\right\|_{-\ell, \Omega_{1}} \leq C_{3} h^{\mu} H^{-a} .
$$


Note that the number $a$ measures how well it is possible to estimate the negativeorder norm of the divided differences of $u-U$. In the worst case, $a=\ell$; this is the case treated by Mock and Lax [21]. In the finite element framework, however, it is possible to take $a$ to be different from $\ell$, as Bramble and Schatz [5] showed for second-order elliptic problems.

Inserting the inequality 3.3 in the inequality of Theorem 3.1 , we get

$$
\begin{aligned}
\left\|u-K_{H}^{\nu, \ell 1} \star U\right\|_{0, \Omega_{0}} & \leq \frac{H^{\nu}}{\nu !} C_{1}|u|_{\nu, \Omega_{1}}+C_{1} C_{2} C_{3} h^{\mu} H^{-a} \\
& \leq C_{1} \max \left\{|u|_{\nu, \Omega_{1}} / \nu !, C_{2} C_{3}\right\}\left(H^{\nu}+h^{\mu} H^{-a}\right) .
\end{aligned}
$$

If we now define $\hat{H}$ to be the solution of the equation $H^{\nu}=h^{\mu} H^{-a}$, we obtain the following result.

Corollary 3.2. Let the hypotheses of Theorem 3.1 hold, and suppose that 3.3 is valid. Then, for $\hat{H}=h^{\mu /(\nu+a)} \leq H_{0}$, we have

$$
\left\|u-K_{\hat{H}}^{\nu, \ell \mathbf{1}} \star U\right\|_{0, \Omega_{0}} \leq C h^{\theta \mu}
$$

where $C=2 C_{1} \max \left\{|u|_{\nu, \Omega_{1}} / \nu !, C_{2} C_{3}\right\}$ and $\theta=\nu /(\nu+a)$.

Note that in the worst possible case, that is when $a=\ell$, this implies that

$$
\left\|u-K_{\hat{H}}^{\nu, \ell \mathbf{1}} \star U\right\|_{0, \Omega_{0}} \leq C h^{\theta \mu}
$$

with $\theta=\nu /(\nu+\ell)<1$. The only possibility we then have for raising the order of convergence is to hope that the function $u$ is very smooth so that we can choose $\nu$ large and positive. Unfortunately, even if this were actually possible, the support of the convolution kernel would be contained in a cube whose diameter is of order $\hat{H}=h^{\mu /(\nu+\ell)}$ which converges to a quantity of order one as $\nu$ increases to infinity; this in turn renders the evaluation of the convolution computationally inefficient.

On the other hand, in the best possible case (that is when $a=0$ ), taking $\nu=\mu$ would permit choosing $\theta=1, \hat{H}=h$ and we would then have

$$
\left\|u-K_{\hat{H}}^{\nu, \ell \mathbf{1}} \star U\right\|_{0, \Omega_{0}} \leq C h^{\mu} .
$$

In other words, for $a=0$ we obtain the same order of convergence for $u-K_{H}^{\nu, \ell \mathbf{1}} \star U$ in the local $L^{2}$-norm as that of the local negative-order norm error estimate in (3.3). Moreover, this is achieved by using a convolution kernel whose support is contained in a cube whose diameter is of the order $\hat{H}=h$ only; this renders the evaluation of the convolution a very fast computation. The examples shown in the Introduction correspond to this case with $\nu=\mu=2 k+1$, where $k$ is the degree of polynomials in the discontinuous Galerkin method. 


\subsection{Negative-order norm error estimates for finite element methods.}

3.2.1. The weak solution. As stated in the Introduction, we consider the following Cauchy problem:

$$
\begin{array}{ll}
u_{t}+\sum_{j=1}^{d} A_{j} u_{x_{j}}+A_{0} u=0, & (x, t) \in \mathbb{R}^{d} \times(0, T], \\
u(x, 0)=u_{0}(x), & x \in \mathbb{R}^{d} .
\end{array}
$$

To make the presentation of the ideas as simple as possible, we reduce inessential technicalities by assuming that the matrices in the equation (3.4) are independent of time and space and by taking the initial data to be 1-periodic in each of the coordinate directions $x_{i}, i=1, \ldots, d$, and we seek a solution to the above problem which is 1-periodic in each coordinate direction.

We suppose that the system of equations (3.4) is strongly hyperbolic, that is, there exists a family of real $m \times m$ matrices $\left\{S(\xi): \xi \in \mathbb{R}^{d}\right\}$ and a constant $K>0$ such that

$$
S(\xi)\left(\sum_{j=1}^{d} A_{j} \xi_{j}\right) S^{-1}(\xi)
$$

is a diagonal matrix for all $\xi \in \mathbb{R}^{d}$, and

$$
\sup _{|\xi|=1}\left(\|S(\xi)\|+\left\|S^{-1}(\xi)\right\|\right) \leq K .
$$

Letting $I=(0,1)^{d}$, the weak solution, $u(x, t)$, of (3.4) satisfies

$$
(u, \varphi)_{I}(t)=\left(u_{0}, \varphi(0)\right)_{I}+\int_{0}^{t}\left(u, \varphi_{t}+\sum_{j=1}^{d} A_{j}^{*} \varphi_{x_{j}}-A_{0}^{*} \varphi\right)_{I} \mathrm{~d} \tau
$$

for all $\varphi \in C^{\infty}\left([0, T] ; H_{\text {per }}^{1}\left(\mathbb{R}^{d}, \mathbb{R}^{m}\right)\right)$ and $t \in[0, T]$, where $A_{j}^{*}$ is the transpose of $A_{j}$ and in the above equation and below

$$
(u, \varphi)_{J}(t)=\int_{J} u(x, t) \varphi(x, t) \mathrm{d} x .
$$

Here, $H_{\text {per }}^{1}\left(\mathbb{R}^{d}, \mathbb{R}^{m}\right)$ denotes the Sobolev space of 1 -periodic functions defined as follows. Let $\mathcal{C}_{\text {per }}^{\infty}\left(\mathbb{R}^{d}, \mathbb{R}^{m}\right)$ be the subset of $\mathcal{C}^{\infty}\left(\mathbb{R}^{d}, \mathbb{R}^{m}\right)$ of 1 -periodic functions. We then define $H_{\text {per }}^{1}\left(\mathbb{R}^{d}, \mathbb{R}^{m}\right)$ as the closure of $\mathcal{C}_{\text {per }}^{\infty}\left(\mathbb{R}^{d}, \mathbb{R}^{m}\right)$ for the $H^{1}\left(I, \mathbb{R}^{m}\right)$-norm.

It follows from (3.6) that the problem (3.7) is well posed in

$$
L_{\text {per }}^{2}\left(\mathbb{R}^{d} ; \mathbb{R}^{m}\right)=\left\{f \in L_{\text {loc }}^{2}\left(\mathbb{R}^{d} ; \mathbb{R}^{m}\right): f(x+\alpha)=f(x) \text { for all } x \in \mathbb{R}^{d}, \alpha \in \mathbb{Z}^{d}\right\}
$$

with respect to the norm $\|\cdot\|_{L^{2}(I)}$; see Theorem 6.3.2. on p. 219 of [14].

3.2.2. The finite element methods. Next, we describe the class of finite element approximations to (3.4). It includes the standard Galerkin method, the Galerkin method with artificial diffusion and the discontinuous Galerkin method. With slight modifications we could have easily included, for example, the streamline-diffusion method and the stabilized discontinuous Galerkin method; however, in order to avoid unnecessary technical complications, we have chosen not to consider these.

Let $\mathcal{T}_{h}=\{K\}$ be a regular triangulation of $\mathbb{R}^{d}$, invariant under translations by $\alpha \in \mathbb{Z}^{d}$, whose elements $K$ are open and have diameter $h_{K}$ less than or equal to 
TABLE 3.1. Examples of finite element methods.

\begin{tabular}{c|c|c}
\hline Method & $\mathcal{M}_{h} \subset \mathcal{C}^{0}$ & $B(U, \eta)$ \\
\hline Standard Galerkin (SG) & yes & $(\mathcal{A} U, \eta)_{I}$ \\
SG with artificial-diffusion & yes & $(\mathcal{A} U, \eta)_{I}+h^{\gamma}(\nabla U, \nabla \eta)_{I}, \gamma \geq 1$ \\
Discontinuous Galerkin (DG) & no & $\left(U, \mathcal{A}^{*} \eta\right)_{h}+\langle\mathcal{A} U, \eta\rangle_{h}$ \\
\hline
\end{tabular}

$h$. It will be assumed throughout that each $K \in \mathcal{T}_{h}$ is contained either in $I$ or in $\mathbb{R}^{d} \backslash I$. For a nonnegative integer $k$, we associate with the triangulation $\mathcal{T}_{h}$ the broken Sobolev space

$$
H_{\mathrm{per}, h}^{k}\left(\mathbb{R}^{d} ; \mathbb{R}^{m}\right)=\Pi_{K \in \mathcal{T}_{h}} H^{k}\left(K ; \mathbb{R}^{m}\right) \cap L_{\mathrm{per}}^{2}\left(\mathbb{R}^{d} ; \mathbb{R}^{m}\right) .
$$

For $k=0$, we shall write $L_{\mathrm{per}, h}^{2}\left(\mathbb{R}^{d} ; \mathbb{R}^{m}\right)=H_{\mathrm{per}, h}^{0}\left(\mathbb{R}^{d} ; \mathbb{R}^{m}\right)$. We then consider two finite element subspaces $\mathcal{M}_{h}$ and $\mathcal{N}_{h}$ of $H_{\mathrm{per}, h}^{1}\left(\mathbb{R}^{d} ; \mathbb{R}^{m}\right)$, and the broken $L_{2}$ inner product $(\cdot, \cdot)_{h}$ defined on $L_{\mathrm{per}, h}^{2}\left(\mathbb{R}^{d} ; \mathbb{R}^{m}\right) \times L_{\mathrm{per}, h}^{2}\left(\mathbb{R}^{d} ; \mathbb{R}^{m}\right)$ by

$$
(W, \chi)_{h}=\sum_{K \in \mathcal{T}_{h, I}}(W, \chi)_{K},
$$

where $\mathcal{T}_{h, I}=\{K \in \mathcal{T}: K \subset I\}$.

We define the finite element approximation $U:[0, T] \rightarrow \mathcal{M}_{h}$ as the solution to

$$
\begin{aligned}
& \left(U_{t}(t), \chi\right)_{h}+B(U(t), \chi)=0, \quad \chi \in \mathcal{N}_{h}, \\
& U(0)=P_{h} u_{0}
\end{aligned}
$$

where $B(\cdot, \cdot)$ is a bilinear form defined on $\mathcal{M}_{h} \times H_{\mathrm{per}, h}^{1}\left(\mathbb{R}^{d} ; \mathbb{R}^{m}\right)$, and the operator $P_{h}: L_{\text {per }}^{2}\left(\mathbb{R}^{d} ; \mathbb{R}^{m}\right) \rightarrow \mathcal{M}_{h}$ is the orthogonal projection in the norm of $L^{2}(I)$.

In Table 3.1 we describe different choices of the form $B$ that give rise to different finite element methods; in each of these $\mathcal{M}_{h}=\mathcal{N}_{h}$, although this need not be the case in general.

The operator $\mathcal{A}$ and the bilinear form $\langle\cdot, \cdot\rangle_{h}$ that appear in Table 3.1 are defined as

$$
\begin{aligned}
\mathcal{A} \chi & =\sum_{j=1}^{d} A_{j} \chi_{x_{j}}+A_{0} \chi \\
\mathcal{A}^{*} \chi & =-\sum_{j=1}^{d} A_{j}^{*} \chi_{x_{j}}+A_{0}^{*} \chi \\
\langle\mathcal{A} U, \chi\rangle_{h} & =\sum_{K \in \mathcal{T}_{h, I}} \sum_{e \in \partial K}\langle\mathcal{A} \cdot n \widehat{U}, \eta\rangle_{e},
\end{aligned}
$$

where $\mathcal{A} \cdot n=A_{1} n_{1}+\cdots+A_{d} n_{d}, n=\left(n_{1}, \ldots, n_{d}\right)$ is the unit outward normal vector to $K$ on $e \subset \partial K$, and $\widehat{U}$ is the numerical flux of the DG method defined as follows. Given an element $K$ and a face $e \in \partial K$, let us denote by $K_{e} \in \mathcal{T}_{h}$ the element sharing the edge $e$ with $K$ and denote by $U_{K}$ and $U_{K_{e}}$ the traces of $U$ on $e$ from $K$ and $K_{e}$, respectively. We compute the $m \times m$ diagonal matrix $\operatorname{diag}\left(\lambda_{1}, \ldots, \lambda_{m}\right)=S(n)(\mathcal{A} \cdot n) S^{-1}(n)$ and set $V=S^{-1}(n) U$ and

$$
\widehat{V}_{j}= \begin{cases}\left(V_{K}\right)_{j} & \text { if } \lambda_{j}>0, \\ \left(V_{K_{e}}\right)_{j} & \text { otherwise. }\end{cases}
$$


The numerical flux is defined as

$$
\widehat{U}=S(n) \widehat{V}
$$

3.2.3. The negative-order error estimate. We now give sufficient conditions for the finite element method which ensure that, for a given time $T$, our approximate solution, $U(T)$, converges with high order in a negative-order norm over a given subdomain $\Omega_{0} \Subset I$ to the weak solution $u(T)$. Given that $l \geq 0$, we wish to estimate

$$
\|u(T)-U(T)\|_{-\ell, \Omega_{0}}=\sup _{\Phi \in \mathcal{C}_{0}^{\infty}\left(\Omega_{0}\right)} \frac{(u(T)-U(T), \Phi)}{\|\Phi\|_{\ell, \Omega_{0}}} .
$$

We begin by considering the solution to the dual problem: Find a function $\varphi$ such that $\varphi(\cdot, t)$ is 1-periodic in each coordinate direction for all $t \in[0, T)$ and

$$
\begin{array}{cl}
\varphi_{t}+\sum_{j=1}^{d} A_{j}^{*} \varphi_{x_{j}}-A_{0}^{*} \varphi=0, & \text { in } \mathbb{R}^{d} \times(0, T), \\
\varphi(x, T)=\Phi(x), & x \in \mathbb{R}^{d},
\end{array}
$$

where $\Phi$ is an arbitrary function in $C_{0}^{\infty}\left(\Omega_{0}\right)$.

$$
\begin{aligned}
(u(T)-U(T), \Phi) & =(u, \varphi)(T)-(U, \varphi)(T) \\
& =\left(u_{0}, \varphi(0)\right)-(U, \varphi)(T) \\
& =\left(u_{0}, \varphi(0)\right)-(U, \varphi)(0)+\int_{0}^{T} \frac{\mathrm{d}}{\mathrm{d} t}(U, \varphi) \mathrm{d} \tau \\
& =\left(u_{0}-P_{h} u_{0}, \varphi(0)\right)-\int_{0}^{T}\left\{\left(U_{t}, \varphi\right)+\left(U, \varphi_{t}\right)\right\} \mathrm{d} \tau .
\end{aligned}
$$

Since, by (3.9), for $\chi:[0, T] \rightarrow \mathcal{N}_{h}$,

$$
\begin{aligned}
\int_{0}^{T}\left(U_{t}, \varphi\right) \mathrm{d} \tau & =\int_{0}^{T}\left(U_{t}, \varphi-\chi\right) \mathrm{d} \tau+\int_{0}^{T}\left(U_{t}, \chi\right) \mathrm{d} \tau \\
& =\int_{0}^{T}\left(U_{t}, \varphi-\chi\right) \mathrm{d} \tau-\int_{0}^{T} B(U, \chi) \mathrm{d} \tau \\
& =\int_{0}^{T}\left\{\left(U_{t}, \varphi-\chi\right)+B(U, \varphi-\chi)\right\} \mathrm{d} \tau-\int_{0}^{T} B(U, \varphi) \mathrm{d} \tau,
\end{aligned}
$$

we obtain that

$$
(u(T)-U(T), \Phi)=\Theta_{M}+\Theta_{N}+\Theta_{C}
$$

where

$$
\begin{aligned}
& \Theta_{M}=\left(u_{0}-P_{h} u_{0}, \varphi(0)\right), \\
& \Theta_{N}=-\int_{0}^{T}\left\{\left(U_{t}, \varphi-\chi\right)+B(U, \varphi-\chi)\right\} \mathrm{d} \tau, \\
& \Theta_{C}=-\int_{0}^{T}\left\{\left(U, \varphi_{t}\right)-B(U, \varphi)\right\} \mathrm{d} \tau .
\end{aligned}
$$

Next, we introduce some general assumptions on $\mathcal{M}_{h}$ and $\mathcal{N}_{h}$ which will enable us to estimate these three terms.

Let $\Omega_{0} \Subset \Omega_{1} \subset I, r \geq 0, \ell \geq 1$, and suppose that $u_{0} \in L_{\text {per }}^{2}\left(\mathbb{R}^{d} ; \mathbb{R}^{m}\right) \cap$ $H^{r}\left(\mathcal{D} \Omega_{1} ; \mathbb{R}^{m}\right)$, where $\mathcal{D} \Omega_{1}$ denotes the domain of dependence for the set $\Omega_{1}$; see Figure 3.1 


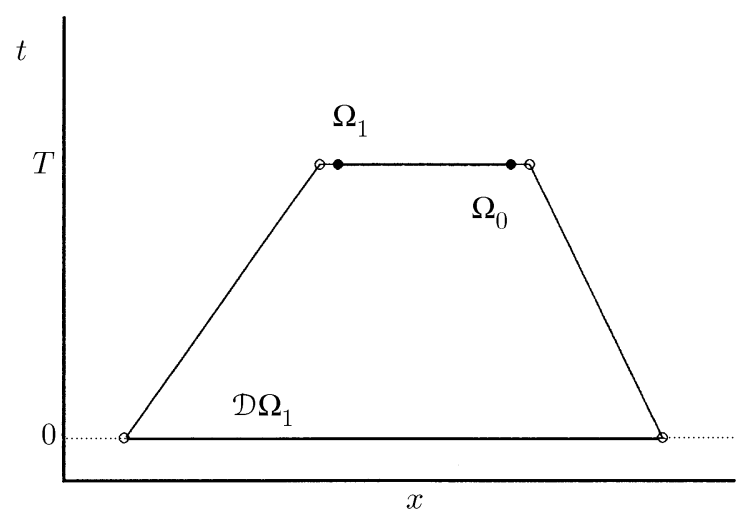

Figure 3.1. Example of the domain of smoothness of $u(T), \Omega_{0}$, and of a domain $\Omega_{1} \ni \Omega_{0}$ and its corresponding domain of dependence $\mathcal{D} \Omega_{1}$.

We adopt the following hypotheses.

(i) Approximation properties of $\mathcal{M}_{h}$ and $P_{h}$. There exist constants $\rho_{M}$, $s_{M}$, with $0 \leq \rho_{M} \leq \ell$ and $0 \leq s_{M} \leq r$, and $\mathbb{A}_{M}$ such that, for each function $\Phi$ in $C_{0}^{\infty}\left(\Omega_{0}\right)$,

$$
\left|\left(u_{0}-P_{h} u_{0}, \varphi(0)\right)\right| \leq \mathbb{A}_{M} h^{\rho_{M}+s_{M}}\left\|u_{0}\right\|_{r, \mathcal{D} \Omega_{1}}\|\Phi\|_{H^{\ell}}
$$

where $\varphi$ is the solution to the dual problem (3.12), (3.13) with final data $\Phi$.

(ii) Residual. Given that $U$ is the solution to (3.9), (3.10), there exist constants $\rho_{N}, s_{N}$, with $0 \leq \rho_{N} \leq \ell$ and $0 \leq s_{N} \leq r$, and $\mathbb{A}_{N}$, such that for each function $\Phi$ in $C_{0}^{\infty}\left(\Omega_{0}\right)$ there exists $\chi \in C^{1}\left([0, T] ; \mathcal{N}_{h}\right)$ with

$\left|\int_{0}^{T}\left(U_{t}, \varphi-\chi\right)_{h}+B(U, \varphi-\chi) \mathrm{d} t\right| \leq \mathbb{A}_{N} h^{\rho_{N}+s_{N}}\left\|u_{0}\right\|_{r, \mathcal{D} \Omega_{1}}\|\Phi\|_{H^{\ell}}$

where $\varphi$ is the solution to the dual problem (3.12), (3.13) with final data $\Phi$.

(iii) Consistency. Given that $U$ is the solution to (3.9), (3.10), there exist constants $s_{C} \in(0, \infty]$ and $\mathbb{A}_{C} \in[0, \infty)$ such that

$$
\left|\int_{0}^{T}\left(U, \varphi_{t}\right)_{h}-B(U, \varphi) \mathrm{d} t\right| \leq \mathbb{A}_{C} h^{s_{C}}\left\|u_{0}\right\|_{r, \mathcal{D} \Omega_{1}}\|\Phi\|_{\ell, \Omega_{0}},
$$

where $\varphi$ is the solution to the dual problem (3.12), 3.13) with final data $\Phi$.

The next result is a trivial consequence of the decomposition (3.14) and conditions (i)-(iii).

Theorem 3.3. Suppose that $u_{0} \in L_{\text {per }}^{2}\left(\mathbb{R}^{d} ; \mathbb{R}^{m}\right) \cap H^{r}\left(\mathcal{D} \Omega_{1} ; \mathbb{R}^{m}\right)$, with $\Omega_{0} \Subset \Omega_{1} \subset I$, $r \geq 0$, and assume that conditions (i)-(iii) hold. Then, for $\ell \geq 1$, we have

$$
\|(u-U)(T)\|_{-\ell, \Omega_{0}} \leq C_{4} h^{s}\left\|u_{0}\right\|_{r, \mathcal{D} \Omega_{1}},
$$

where $s=\min \left\{\rho_{M}+s_{M}, \rho_{N}+s_{N}, s_{C}\right\}$ and $C_{4}=\mathbb{A}_{M}+\mathbb{A}_{N}+\mathbb{A}_{C}$. 
TABLE 3.2. The parameters of Theorem 3.3 for finite element methods using piecewise polynomials of degree $k$.

\begin{tabular}{c|ccc}
\hline parameter & $\mathrm{SG}$ & $\mathrm{SG}$ with $\mathrm{AD}$ & $\mathrm{DG}$ \\
\hline$\rho_{M}$ & $\min \{k+1, \ell\}$ & $\min \{k+1, \ell\}$ & $\min \{k+1, \ell\}$ \\
$s_{M}$ & $\min \{k+1, r\}$ & $\min \{k+1, r\}$ & $\min \{k+1, r\}$ \\
$\rho_{N}$ & $\min \{k, \ell\}$ & $\min \{k, \ell\}$ & $\min \{k+1 / 2, \ell\}$ \\
$s_{N}$ & $\min \{k, r\}$ & $\min \{k, r\}$ & $\min \{k+1 / 2, r\}$ \\
$s_{C}$ & $\infty$ & $\gamma$ & $\infty$ \\
\hline
\end{tabular}

In Table 3.2 we display the parameters of the above result for some finite element methods; for each of the methods listed we have $\mathcal{M}_{h}=\mathcal{N}_{h}$ and have assumed that $\Omega_{1}=I$ (so that $\mathcal{D} \Omega_{1}=I$ also).

3.3. The error estimates. Now we combine the results obtained in the previous subsections.

Theorem 3.4. Let $u$ be the exact solution of problem (3.4), 3.5 and let $U$ be the approximation defined by (3.9), (3.10) for which conditions (i)-(iii) are valid. Consider the convolution kernel $K_{\hat{H}}^{\nu, \ell 1}$ of Theorem 3.1 Let each of the components of $u(T)$ be in $H^{\nu}\left(\Omega_{1}\right)$ and let $\Omega_{0}$ be such that $\Omega_{0}+2 \operatorname{supp}\left(K_{\hat{H}}^{\nu, \ell \mathbf{1}}\right) \Subset \Omega_{1}$. Then, for general regular triangulations and $\hat{H}=h^{s /(\nu+\ell)} \leq H_{0}$, we have

$$
\left\|u(T)-K_{\hat{H}}^{\nu, \ell 1} \star U(T)\right\|_{0, \Omega_{0}} \leq C h^{\theta s},
$$

where $\theta, s$ and $C$ are as in Theorem 3.3 with $C_{3}=C_{4}\left\|u_{0}\right\|_{r, \mathcal{D} \Omega_{1}}$ and $\theta=\nu /(\nu+\ell)$. Moreover, if the triangulation is translation invariant on a neighborhood of the support of the solution of the adjoint equation (3.12), (3.13), then, for $\hat{H}=h$,

$$
\left\|u(T)-K_{\hat{H}}^{\nu, \ell 1} \star U(T)\right\|_{0, \Omega_{0}} \leq C h^{s},
$$

$C_{3}=C_{4}\left\|u_{0}\right\|_{r+\ell, \mathcal{D} \Omega_{1}}$.

Proof. The first inequality is a direct consequence of Corollary 3.2 and Theorem 3.3. The second inequality also follows from the above results and from the fact that if the triangulation is translation invariant in a neighborhood (of order $\hat{H}=h$ ) of the support of the solution of the adjoint problem, then we have

$$
\left\|\partial_{\hat{H}}^{\alpha}(u-U)(T)\right\|_{-\ell, \Omega_{0}} \leq C_{4} h^{s}\left\|\partial_{\hat{H}}^{\alpha} u_{0}\right\|_{r, \mathcal{D} \Omega_{1}} .
$$

This completes the proof.

TABLE 3.3. Orders of convergence with piecewise polynomials of degree $k$ when the analytical solution $u$ is in $C\left([0, T] ; H_{\text {per }}^{\nu}(I)\right)$.

\begin{tabular}{c|c|ccc}
\hline triangulations & $\nu$ & SG & SG with AD & DG \\
\hline general & 0 & $\theta k$ & $\theta \alpha$ & $\theta(k+1 / 2)$ \\
general & $2 k+2$ & $\theta k$ & $\theta \alpha$ & $\theta(k+1 / 2)$ \\
locally invariant & 0 & $k$ & $\alpha$ & $k+1 / 2$ \\
locally invariant & $2 k+2$ & $2 k$ & $\alpha$ & $2 k+1$ \\
\hline
\end{tabular}


Some important particular cases for which $\Omega_{1}=I$ (and consequently $\mathcal{D} \Omega_{1}=I$ ) are collected in Table 3.3, in fact these are the estimates we can actually prove. The case in which $\Omega_{1} \neq I$ remains a challenging open problem.

\section{Proofs}

4.1. The approximation result. In this subsection, for the sake of completeness, we sketch the proof of Theorem 3.1] we follow Bramble and Schatz [5].

Consider the quantity

$$
\Theta_{H}=\left\|u-K_{H}^{\nu, \ell 1} \star U\right\|_{0, \Omega_{0}} \leq \Theta_{H, 1}+\Theta_{H, 2}
$$

where

$$
\begin{aligned}
& \Theta_{H, 1}=\left\|u-K_{H}^{\nu, \ell 1} \star u\right\|_{0, \Omega_{0}}, \\
& \Theta_{H, 2}=\left\|K_{H}^{\nu, \ell 1} \star(u-U)\right\|_{0, \Omega_{0}} .
\end{aligned}
$$

To estimate $\Theta_{H, 1}$, we denote by $\mathcal{I}$ the support of $K^{(\nu, \ell \mathbf{1})}$, we label by $T^{\nu} u(y, \cdot)$ the Taylor polynomial of degree $\nu-1$ of $u$ around $y$, and put $\mathcal{R}^{\nu} u(y, \cdot)=u(\cdot)-T^{\nu} u(y, \cdot)$. We then easily deduce that

$$
u(x)-K_{H}^{\nu, \ell 1} \star u(x)=R^{\nu} u(y, x)-\int_{\mathcal{I}} K^{\nu, \ell 1}(z) R^{\nu} u(y, x-H z) d z
$$

by using the fact that the kernel $K_{H}^{\nu, \ell \mathbf{1}}$ reproduces polynomials of degree $\nu-1$ by convolution. For $y=x$, the above expression becomes

$$
u(x)-K_{H}^{\nu, \ell 1} \star u(x)=-\int_{\mathcal{I}} K^{\nu, \ell 1}(z) R^{\nu} u(x, x-H z) \mathrm{d} z,
$$

and we obtain

$$
\begin{aligned}
\Theta_{H, 1} & \leq\left\|K^{\nu, \ell \mathbf{1}}\right\|_{L^{1}\left(\mathbb{R}^{d}\right)} \sup _{z \in \mathcal{I}}\left\|R^{\nu} u(\cdot, \cdot-H z)\right\|_{0, \Omega_{0}} \\
& \leq \frac{H^{\nu}}{\nu !}\left\|K^{\nu, \ell \mathbf{1}}\right\|_{L^{1}(\mathcal{I})}|u|_{\nu, \Omega_{0}+H \mathcal{I}} .
\end{aligned}
$$

On applying the triangle inequality to the expression of $K^{\nu, \ell 1}$ given by (3.1), we get

$$
\left\|K^{\nu, \ell 1}\right\|_{L^{1}(\mathcal{I})} \leq \sum_{\gamma \in \mathbb{Z}^{d}}\left|k_{\gamma}^{\nu, \ell 1}\right|\left\|\psi^{\ell \mathbf{1}-\alpha}(\cdot-\gamma)\right\|_{L^{1}\left(\mathbb{R}^{d}\right)}=\sum_{\gamma \in \mathbb{Z}^{d}}\left|k_{\gamma}^{\nu, \ell 1}\right|=C_{1},
$$

since $\left\|\psi^{\ell \mathbf{1}-\alpha}(\cdot-\gamma)\right\|_{L^{1}\left(\mathbb{R}^{d}\right)}=1$. This implies that

$$
\Theta_{H, 1} \leq \frac{H^{\nu}}{\nu !} C_{1}|u|_{\nu, \Omega_{0}+H \mathcal{I}} \leq \frac{H^{\nu}}{\nu !} C_{1}|u|_{\nu, \Omega_{1}} .
$$

Now, let us sketch the procedure to estimate $\Theta_{h, 2}$. Take a set $\Omega_{1 / 2}$ such that, for all $H \leq H_{0}$,

$$
\begin{aligned}
& \Omega_{0}+\operatorname{supp}\left(K_{h}^{\nu, \ell}\right) \subset \Omega_{1 / 2}, \\
& \Omega_{1 / 2}+\operatorname{supp}\left(K_{h}^{\nu, \ell}\right) \subset \Omega_{1} .
\end{aligned}
$$

Then, setting $e(x)=u(x)-U(x)$, we get

$$
\Theta_{H, 2}=\left\|K_{H}^{\nu, \ell 1} \star e\right\|_{0, \Omega_{0}} \leq C_{2} \sum_{|\alpha| \leq \ell}\left\|D^{\alpha}\left(K_{H}^{\nu, \ell 1} \star e\right)\right\|_{-\ell, \Omega_{1 / 2}}
$$


where $C_{2}$ depends solely on $\Omega_{0}, \Omega_{1 / 2}, d, \nu$, and $\ell$, by Lemma 4.2 in Bramble and Schatz [5]. This is the significant step that allows us to pass from the $L^{2}$-norm to a negative-order Sobolev norm.

Next, we exploit the fact that the kernel $K_{H}^{\nu, \ell \mathbf{1}}$ is a linear combination of B-splines given by (3.1); this is the only place in this proof where properties of B-splines are used. Thus, by the property (3.2), we have that

$$
D^{\alpha}\left(K_{H}^{\nu, \ell 1} \star e\right)=K_{H}^{\nu, \ell 1 ; \alpha} \star \partial_{H}^{\alpha} e,
$$

where

$$
K^{\nu, \ell \mathbf{1} ; \alpha}(y)=\sum_{\gamma \in \mathbb{Z}^{d}} k_{\gamma}^{\nu, \ell \mathbf{1}} \psi^{(\ell \mathbf{1}-\alpha)}(y-\gamma)
$$

This implies that

$$
\Theta_{H, 2} \leq C_{2} \sum_{|\alpha| \leq \ell}\left\|K_{H}^{\nu, \ell \mathbf{1} ; \alpha} \star \partial_{H}^{\alpha} e\right\|_{-\ell, \Omega_{1 / 2}} \leq C_{2} \sum_{|\alpha| \leq \ell}\left\|K_{H}^{\nu, \ell \mathbf{1} ; \alpha}\right\|_{L^{1}\left(\mathbb{R}^{d}\right)}\left\|\partial_{H}^{\alpha} e\right\|_{-\ell, \Omega_{1}} .
$$

Finally, since $\left\|K_{H}^{\nu, \ell \mathbf{1} ; \alpha}\right\|_{L^{1}\left(\mathbb{R}^{d}\right)}=\left\|K^{\nu, \ell \mathbf{1} ; \alpha}\right\|_{L^{1}\left(\mathbb{R}^{d}\right)} \leq C_{1}$, we get

$$
\Theta_{H, 2} \leq C_{1} C_{2} \sum_{|\alpha| \leq \ell}\left\|\partial_{H}^{\alpha} e\right\|_{-\ell, \Omega_{1}}
$$

This completes the proof of Theorem 3.1

4.2. The conditions (i)-(iii) for some finite element methods. In this subsection, we justify the results displayed in Table 3.2 .

a. The SG method. Let us begin by considering property (i). For the $L^{2}$ projection, it is well known that $\rho_{M}=\min \{k+1, \ell\}$ and that $s_{M}=\min \{k+1, r\}$ for regular triangulations. Next, let us consider property (iii). A simple calculation shows that we can take $\mathbb{A}^{C}=0$; this allows us to take $s_{C}=\infty$.

For property (ii), we proceed as follows:

$$
\begin{aligned}
\Theta & =\int_{0}^{T}\left\{\left(U_{t}, \varphi-\chi\right)_{h}+B(U, \varphi-\chi)\right\} \mathrm{d} t \\
& =\int_{0}^{T}\left\{\left((U-u)_{t}+\mathcal{A}(U-u), \varphi-\chi\right)_{h}\right\} \mathrm{d} t .
\end{aligned}
$$

Taking $\chi$ as the $L^{2}$-projection of $\varphi$, we get (ii) with $\rho_{N}=\min \{k, \ell\}, s_{N}=\min \{k, r\}$ and $\Omega_{1}=I$.

b. The SG with artificial diffusion. For this method, we only have to focus on property (iii). We have,

$$
\begin{aligned}
\Theta & =\int_{0}^{T}\left\{\left(U, \varphi_{t}\right)_{h}-B(U, \varphi)\right\} \mathrm{d} t=\int_{0}^{T} h^{\gamma}(\nabla U, \nabla \varphi) \mathrm{d} t \\
& \leq h^{\gamma}\|\nabla U\|_{L^{2}\left(0, T ; L^{2}(I)\right)}\|\nabla \varphi\|_{L^{2}\left(0, T ; L^{2}(I)\right)} .
\end{aligned}
$$

This means that property (iii) is satisfied with $s_{C}=\gamma$.

c. The DG method. For properties (i) and (iii), we proceed as in the two methods discussed above. The verification of property (ii) requires a more delicate argument. For a function $W$ whose components are in $H_{\mathrm{per}, h}^{1}\left(\mathbb{R}^{d} ; \mathbb{R}^{m}\right)$, we set 
$\llbracket \mathcal{A} W \rrbracket(x)=\mathcal{A} \cdot n^{+} W^{+}(x)+\mathcal{A} \cdot n^{-} W^{-}(x)$ for every $x \in e$, where $W^{ \pm}(x)=$ $\lim _{z \downarrow 0} W\left(x-z n^{ \pm}\right)$and $n^{ \pm}$is orthogonal to the face $e$ of the element $K$ at $x$. With this notation, we can write that

$$
\begin{aligned}
\Theta & =\int_{0}^{T}\left\{\left(U_{t}, \varphi-\chi\right)_{h}+B(U, \varphi-\chi)\right\} \mathrm{d} t \\
& =\int_{0}^{T}\left\{\left(U_{t}+\mathcal{A} U, \varphi-\chi\right)_{h}+\sum_{e \in \mathcal{E}}\langle\llbracket \mathcal{A} U \rrbracket, \varphi-\chi\rangle_{e}\right\} \mathrm{d} t
\end{aligned}
$$

where we obtained the last step after a simple integration by parts; by $\mathcal{E}$ we denote the collection of faces $e$ of the elements $K$ of the triangulation $\mathcal{T}_{h, I}$.

Now, taking $\chi$ to be the $L^{2}$-projection of $\varphi$ onto $\mathcal{M}_{h}=\mathcal{N}_{h}$, we get

$$
\begin{aligned}
\Theta & =\int_{0}^{T}\left\{\sum_{e \in \mathcal{E}}\langle\llbracket \mathcal{A} U \rrbracket, \varphi-\chi\rangle_{e}\right\} \mathrm{d} t \\
& \leq\left\{\int_{0}^{T} \sum_{e \in \mathcal{E}}\|\llbracket \mathcal{A} U \rrbracket\|_{e, 0}^{2} \mathrm{~d} t\right\}^{1 / 2}\left\{\int_{0}^{T} \sum_{e \in \mathcal{E}}\|\varphi-\chi\|_{e, 0}^{2} \mathrm{~d} t\right\}^{1 / 2} .
\end{aligned}
$$

This implies that property (ii) is satisfied with $s_{N}=\min \{k+1 / 2, \ell\}$ for regular triangulations. It remains to obtain an estimate of the first term on the right; following Johnson and Pitkäranta [16] or Cockburn [7, it is easy to prove that (iii) is satisfied with $\rho_{N}=\min \{k+1 / 2, r\}$ and $\Omega_{1}=I$.

\section{NUMERICAL EXPERIMENTS}

In this section, we validate our theoretical results with an emphasis on the case in which the doubling of the order of convergence is achieved. We also explore the performance of the post-processing technique in situations not predicted by our analysis; thus, we display the $L^{\infty}$-errors in all our experiments, include an example dealing with a linear convection-diffusion equation and an example of a nonlinear convection equation.

We consider the discontinuous Galerkin method with polynomials of degree $k$ and use a third order Runge-Kutta method to discretize in time; the time step $\Delta t$ is chosen small enough so that spatial errors dominate. Results for $P^{1}$ to $P^{4}$ are shown.

The $L^{\infty}$ error measures the maximum numerical error at the six Gaussian points in each element for all elements. The $L^{2}$-error is computed by the six-point Gaussian rule in each element.

Example 5.1. A linear scalar convection equation with smooth solution on the domain $I=[0,2 \pi)$ :

$$
u_{t}+u_{x}=0, \quad \text { in } I \times(0, T), \quad u(x, 0)=\sin (x) \quad x \in I,
$$

with periodic boundary conditions. The errors are computed at $T=12.5$ which is about two periods in time.

In Table 5.1, we show the numerical errors for this problem. We can clearly see that both the $L^{2}$ - and $L^{\infty}$-error for $P^{k}$-elements is of $(k+1)$-th order before postprocessing and of at least $(2 k+1)$-th order after post-processing. This is consistent with our theoretical results. 
TABLE 5.1. Example 5.1, $u_{t}+u_{x}=0$, smooth solution.

\begin{tabular}{|c|c|c|c|c|c|c|c|c|}
\hline & \multicolumn{4}{|c|}{ Before post-processing } & \multicolumn{4}{|c|}{ After post-processing } \\
\hline mesh & $L^{2}$-error & order & $L^{\infty}$-error & order & $L^{2}$-error & order & $L^{\infty}$-error & order \\
\hline \multicolumn{9}{|c|}{$P^{1}$} \\
\hline 10 & $3.29 \mathrm{E}-02$ & - & $5.81 \mathrm{E}-02$ & - & $3.01 \mathrm{E}-02$ & - & $4.22 \mathrm{E}-02$ & $\bar{Z}$ \\
\hline 20 & $5.63 \mathrm{E}-03$ & 2.55 & $1.06 \mathrm{E}-02$ & 2.45 & $3.84 \mathrm{E}-03$ & 2.97 & $5.44 \mathrm{E}-03$ & 2.96 \\
\hline 40 & $1.16 \mathrm{E}-03$ & 2.28 & $2.89 \mathrm{E}-03$ & 1.88 & $4.79 \mathrm{E}-04$ & 3.00 & $6.78 \mathrm{E}-04$ & 3.01 \\
\hline 80 & $2.72 \mathrm{E}-04$ & 2.09 & $8.08 \mathrm{E}-04$ & 1.84 & $5.97 \mathrm{E}-05$ & 3.00 & $8.45 \mathrm{E}-05$ & 3.00 \\
\hline 160 & $6.68 \mathrm{E}-05$ & 2.03 & $2.13 \mathrm{E}-04$ & 1.93 & $7.45 \mathrm{E}-06$ & 3.00 & $1.05 \mathrm{E}-05$ & 3.00 \\
\hline 320 & $1.66 \mathrm{E}-05$ & 2.01 & $5.45 \mathrm{E}-05$ & 1.96 & $9.30 \mathrm{E}-07$ & 3.00 & $1.32 \mathrm{E}-06$ & 3.00 \\
\hline \multicolumn{9}{|c|}{$P^{2}$} \\
\hline 10 & $8.63 \mathrm{E}-04$ & - & $2.86 \mathrm{E}-03$ & - & $2.52 \mathrm{E}-04$ & - & $3.57 \mathrm{E}-04$ & - \\
\hline 20 & $1.07 \mathrm{E}-04$ & 3.01 & $3.69 \mathrm{E}-04$ & 2.95 & $5.96 \mathrm{E}-06$ & 5.40 & $8.41 \mathrm{E}-06$ & 5.41 \\
\hline 40 & $1.34 \mathrm{E}-05$ & 3.00 & $4.63 \mathrm{E}-05$ & 3.00 & $1.53 \mathrm{E}-07$ & 5.29 & $2.16 \mathrm{E}-07$ & 5.28 \\
\hline 80 & $1.67 \mathrm{E}-06$ & 3.00 & $5.78 \mathrm{E}-06$ & 3.00 & $4.22 \mathrm{E}-09$ & 5.18 & $5.97 \mathrm{E}-09$ & 5.18 \\
\hline 160 & $2.09 \mathrm{E}-07$ & 3.00 & $7.23 \mathrm{E}-07$ & 3.00 & $1.27 \mathrm{E}-10$ & 5.06 & $1.80 \mathrm{E}-10$ & 5.06 \\
\hline \multicolumn{9}{|c|}{$P^{3}$} \\
\hline 10 & $3.30 \mathrm{E}-05$ & - & $9.59 \mathrm{E}-05$ & - & $1.64 \mathrm{E}-05$ & - & $2.31 \mathrm{E}-05$ & - \\
\hline 20 & $2.06 \mathrm{E}-06$ & 4.00 & $6.07 \mathrm{E}-06$ & 3.98 & 7.07E-08 & 7.85 & $1.00 \mathrm{E}-07$ & 7.85 \\
\hline 40 & $1.29 \mathrm{E}-07$ & 4.00 & $3.80 \mathrm{E}-07$ & 4.00 & $2.91 \mathrm{E}-10$ & 7.92 & $4.15 \mathrm{E}-10$ & 7.91 \\
\hline 50 & $5.29 \mathrm{E}-08$ & 4.00 & $1.56 \mathrm{E}-07$ & 4.00 & $5.03 \mathrm{E}-11$ & 7.87 & $7.24 \mathrm{E}-11$ & 7.83 \\
\hline \multicolumn{9}{|c|}{$P^{4}$} \\
\hline 10 & $1.02 \mathrm{E}-06$ & - & $2.30 \mathrm{E}-06$ & - & $1.98 \mathrm{E}-06$ & - & $2.81 \mathrm{E}-06$ & 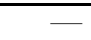 \\
\hline 20 & $3.21 \mathrm{E}-08$ & 5.00 & $7.30 \mathrm{E}-08$ & 4.98 & $2.20 \mathrm{E}-09$ & 9.82 & $3.11 \mathrm{E}-09$ & 9.82 \\
\hline 30 & $4.23 \mathrm{E}-09$ & 5.00 & $9.66 \mathrm{E}-09$ & 4.99 & $4.34 \mathrm{E}-11$ & 9.68 & $6.66 \mathrm{E}-11$ & 9.48 \\
\hline
\end{tabular}
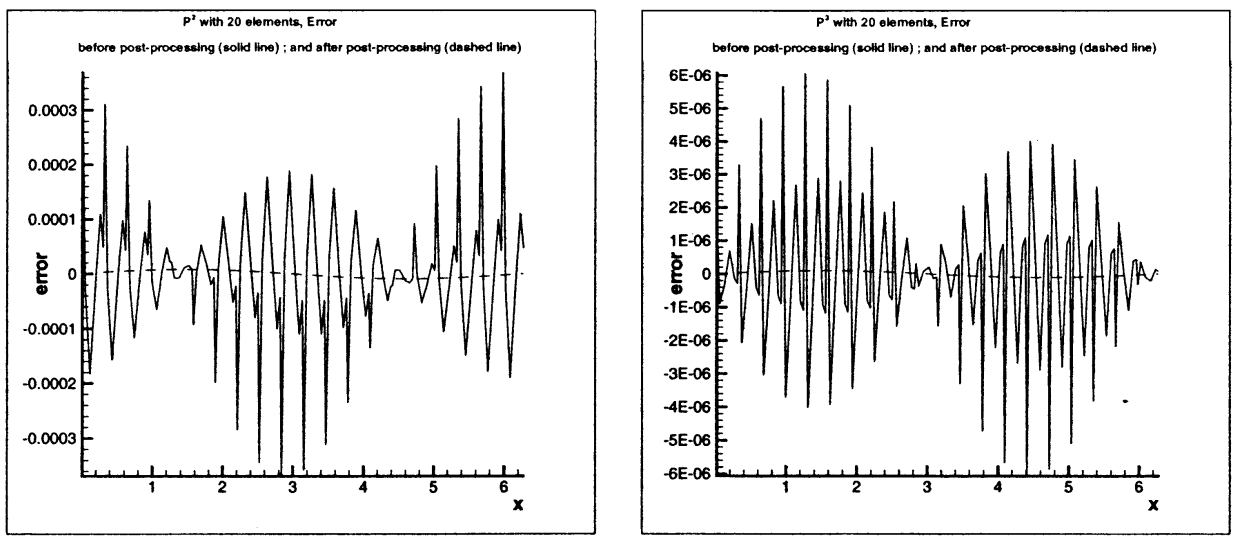

FiguRE 5.1. The errors before and after post-processing for 20 elements: $P^{2}$ (left) and $P^{3}$ (right).

In Figure 5.1 we plot the errors of the numerical solution before and after postprocessing for $P^{2}$ and $P^{3}$ with 20 elements. We can clearly see that the errors before post-processing are highly oscillatory, and the post-processing gets rid of the oscillation in the error and greatly reduces its magnitude. 

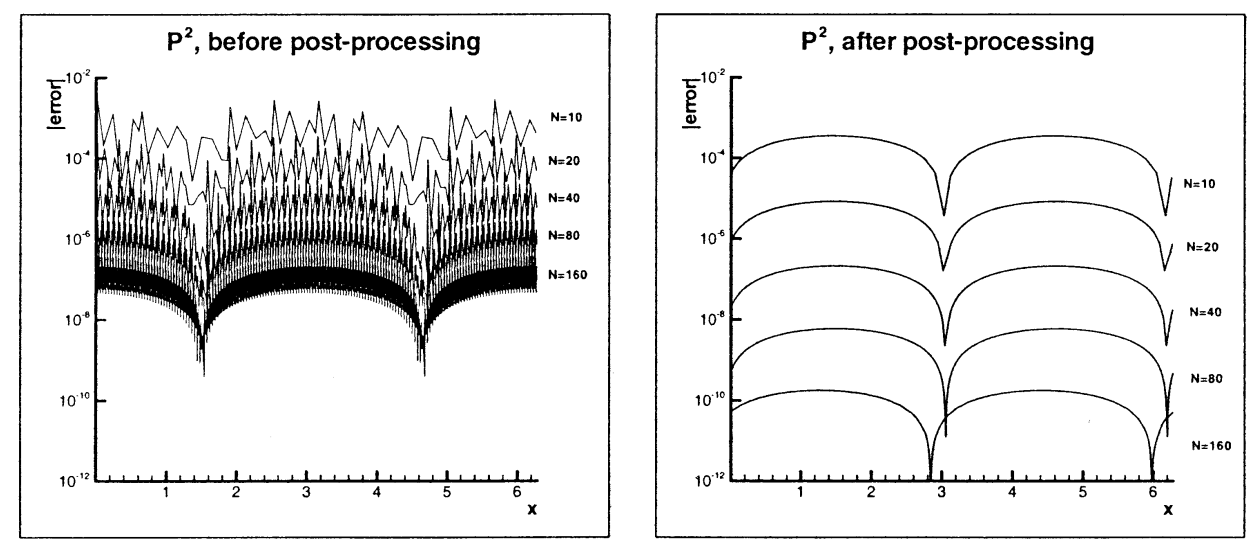

Figure 5.2. The errors in absolute value and in logarithmic scale, for $P^{2}$ with $\mathrm{N}=10,20,40,40,80$ and 160 elements: before postprocessing (left) and after post-processing (right).

In Figure 5.2 we plot the errors, in absolute value and in logarithmic scale, of the numerical solution before and after post-processing for $P^{2}$, with 10, 20, 40, 80 and 160 elements. We can clearly see that the post-processed errors are less oscillatory and much smaller in magnitude, and approximately third- and fifth-order accuracy for the preprocessed and post-processed errors, respectively, measured by the spacing between the errors when the number of elements doubles.

Example 5.2. A linear scalar convection-diffusion equation with smooth solution on the domain $I=[0,2 \pi)$ :

$$
u_{t}+u_{x}=u_{x x}, \quad \text { in } I \times(0, T), \quad u(x, 0)=\sin (x), \quad x \in I,
$$

with periodic boundary conditions. The errors are computed at $T=2$, using the local discontinuous Galerkin method [9], with alternating left and right fluxes for $u$ and the auxiliary variable $q$ which approximates $u_{x}$ (formula (2.9) in [9]).

Although not proven in this paper, we expect the same accuracy result to hold for the post-processed solution as in Example 5.1. In Table 5.2 we show the results for this problem. We can clearly see that the $L^{\infty}$-errors for $P^{k}$-elements are of $(k+1)$-th order before post-processing and of at least $(2 k+1)$-th order after post-processing, both for the solution $u$ and for the auxiliary variable $q$ which approximates $u_{x}$. The results for the $L^{2}$-errors are similar and are not shown to save space.

Example 5.3. The same linear scalar convection equation (5.1) with the same initial condition, except that now $I=[0,5)$. The solution now has a discontinuity at $x=0$ (or $x=5$ ) and this discontinuity moves in time with the characteristic speed 1 . We compute the errors at $T=12.5$, that is, after 2.5 periods in time. The discontinuity at this time is located at $x=2.5$. The errors shown in Table 5.3 are calculated within the "smooth region" $[0,1] \cup[4,5]$, as distance 1.5 away from the discontinuity, namely excluding the interval $1<x<4$. 
TABLE 5.2. Example 5.2, $u_{t}+u_{x}=u_{x x}$, smooth solution.

\begin{tabular}{|c|c|c|c|c|c|c|c|c|}
\hline & \multicolumn{4}{|c|}{ Before post-processing } & \multicolumn{4}{|c|}{ After post-processing } \\
\hline & \multicolumn{2}{|c|}{$u$} & \multicolumn{2}{|c|}{$q$ for $u_{x}$} & \multicolumn{2}{|l|}{$u$} & \multicolumn{2}{|c|}{$q$ for $u_{x}$} \\
\hline mesh & $L^{2}$-error & order & $L^{\infty}$-error & order & $L^{2}$-error & order & $L^{\infty}$-error & order \\
\hline \multicolumn{9}{|c|}{$\overline{P^{1}}$} \\
\hline$\overline{10}$ & $4 \mathrm{E}-03$ & $=$ & $82 \mathrm{E}-23$ & - & $1.19 \mathrm{E}-03$ & - & $1.18 \mathrm{E}-03$ & - \\
\hline 20 & $32 \mathrm{E}-03$ & 1.89 & $71 \mathrm{E}-03$ & 1.77 & $34 \mathrm{E}-04$ & 3.15 & $41 \mathrm{E}-04$ & 3.07 \\
\hline 40 & $68 \mathrm{E}-04$ & 1.96 & $56 \mathrm{E}-04$ & 1.91 & $56 \mathrm{E}-05$ & 3.05 & $69 \mathrm{E}-05$ & 3.06 \\
\hline 80 & $1.19 \mathrm{E}-04$ & 1.98 & $1.17 \mathrm{E}-04$ & 1.96 & $1.46 \mathrm{E}-06$ & 3.02 & $2.07 \mathrm{E}-06$ & $\overline{3.03}$ \\
\hline$\overline{160}$ & $3.00 \mathrm{E}-05$ & 1.99 & $2.98 \mathrm{E}-05$ & 1.98 & $2.32 \mathrm{E}-07$ & 3.03 & $2.57 \mathrm{E}-07$ & 3.01 \\
\hline 320 & $7.52 \mathrm{E}-06$ & 1.99 & $7.50 \mathrm{E}-06$ & 1.99 & $2.87 \mathrm{E}-08$ & 3.01 & $3.20 \mathrm{E}-08$ & 3.01 \\
\hline \multicolumn{9}{|c|}{$P^{2}$} \\
\hline 10 & $97 \mathrm{E}-04$ & - & $3.38 \mathrm{E}-04$ & E- & $2.93 \mathrm{E}-05$ & - & $2.96 \mathrm{E}-05$ & - \\
\hline 20 & $5.01 \mathrm{E}-05$ & 2.99 & $4.61 \mathrm{E}-05$ & 2.87 & -07 & 5.75 & $5.46 \mathrm{E}-07$ & 5.76 \\
\hline$\overline{40}$ & $6.25 \mathrm{E}-06$ & 3.00 & $6.02 \mathrm{E} .06$ & 2.94 & -08 & 5.71 & $1.05 \mathrm{E}-08$ & 5.70 \\
\hline 80 & $7.83 \mathrm{E}-07$ & 3.00 & $7.68 \mathrm{E}-07$ & 2.97 & -10 & 5.57 & $2.26 \mathrm{E}-10$ & $\overline{5.54}$ \\
\hline 160 & $9.78 \mathrm{E}-08$ & 3.00 & $9.69 \mathrm{E}-08$ & 2.99 & $5.31 \mathrm{E}-12$ & 5.37 & $5.63 \mathrm{E}-12$ & $\overline{5.33}$ \\
\hline \multicolumn{9}{|c|}{$P^{3}$} \\
\hline 10 & $30 \mathrm{E}-05$ & E & $13 \mathrm{E}-05$ & - & $3.09 \mathrm{E}-06$ & E & $3.09 \mathrm{E}-06$ & \\
\hline 20 & $8.23 \mathrm{E}-07$ & 3.98 & $7.73 \mathrm{E}-07$ & 3.86 & $1.32 \mathrm{E}-08$ & 7.87 & $1.32 \mathrm{E}-08$ & 7.87 \\
\hline 40 & $5.14 \mathrm{E}-08$ & 4.00 & $4.99 \mathrm{E}-08$ & 3.95 & $5.31 \mathrm{E}-11$ & 7.96 & $5.31 \mathrm{E}-11$ & 7.96 \\
\hline \multicolumn{9}{|c|}{$P^{4}$} \\
\hline 10 & $3.11 \mathrm{E}-07$ & 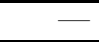 & $2.93 \mathrm{E}-07$ & - & $3.79 \mathrm{E}-07$ & E & $3.80 \mathrm{E}-07$ & 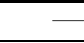 \\
\hline 20 & $9.89 \mathrm{E}-09$ & 4.97 & $9.54 \mathrm{E}-09$ & 4.94 & $4.19 \mathrm{E}-10$ & 9.82 & $4.19 \mathrm{E}-10$ & 9.82 \\
\hline 30 & $1.30 \mathrm{E}-09$ & 5.00 & $1.27 \mathrm{E}-09$ & 4.98 & $7.11 \mathrm{E}-12$ & 10.05 & $7.11 \mathrm{E}-12$ & $\overline{0.0}$ \\
\hline
\end{tabular}
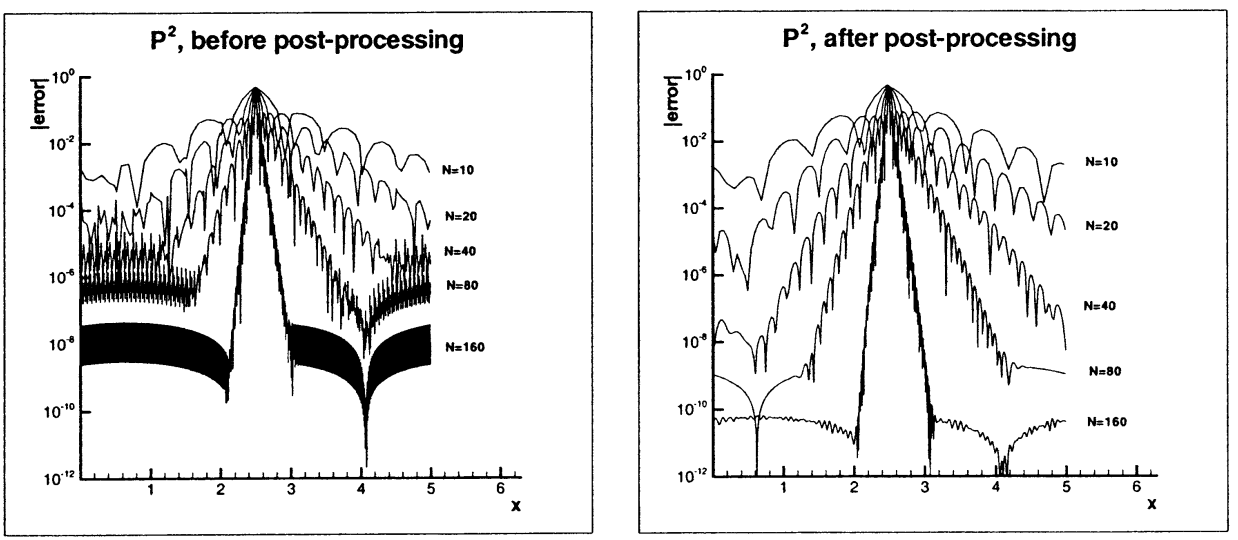

Figure 5.3. The errors in absolute value and in logarithmic scale for $P^{2}$ with $\mathrm{N}=10,20,40,40,80$ and 160 elements: before postprocessing (left) and after post-processing (right).

The theory in this paper would only guarantee $(k+1)$-th order accuracy for $P^{k}$-elements after post-processing since our estimates hold for $\mathcal{D} \Omega_{1}=I$ only and the initial condition displays a discontinuity. However, Table 5.3 shows that both 
TABLE 5.3. Example 5.3, $u_{t}+u_{x}=0$, discontinuous solution, errors in smooth regions

\begin{tabular}{|c|c|c|c|c|c|c|c|c|}
\hline & \multicolumn{4}{|c|}{ Before post-processing } & \multicolumn{4}{|c|}{ After post-processing } \\
\hline mesh & $L^{2}$-error & order & $L^{\infty}$-error & order & $L^{2}$-error & order & $L^{\infty}$-error & order \\
\hline \multicolumn{9}{|c|}{$P^{1}$} \\
\hline 10 & $2.02 \mathrm{E}-02$ & E & $6.46 \mathrm{E}-02$ & E & $1.76 \mathrm{E}-02$ & 二 & $2.80 \mathrm{E}-02$ & - \\
\hline 20 & \begin{tabular}{|l}
$4.37 \mathrm{E}-03$ \\
\end{tabular} & 2.21 & $1.21 \mathrm{E}-02$ & 2.41 & $3.96 \mathrm{E}-03$ & 2.15 & $1.18 \mathrm{E}-02$ & 1.24 \\
\hline 40 & \begin{tabular}{|l|}
$6.63 \mathrm{E}-04$ \\
\end{tabular} & 2.72 & $1.89 \mathrm{E}-03$ & 2.69 & $2.69 \mathrm{E}-04$ & 3.88 & $6.77 \mathrm{E}-04$ & 4.12 \\
\hline 80 & $1.58 \mathrm{E}-04$ & 2.07 & $5.24 \mathrm{E}-04$ & 1.85 & $2.78 \mathrm{E}-05$ & 3.27 & $4.31 \mathrm{E}-05$ & 3.97 \\
\hline 160 & \begin{tabular}{|l|}
$3.92 \mathrm{E}-05$ \\
\end{tabular} & 2.01 & $1.36 \mathrm{E}-04$ & 1.94 & $3.49 \mathrm{E}-06$ & 3.00 & $5.31 \mathrm{E}-06$ & 3.02 \\
\hline 320 & \begin{tabular}{|l}
$9.80 \mathrm{E}-06$ \\
\end{tabular} & 2.00 & $3.47 \mathrm{E}-05$ & 1.97 & $4.37 \mathrm{E}-07$ & 3.00 & $6.63 \mathrm{E}-07$ & 3.00 \\
\hline \multicolumn{9}{|c|}{$P^{2}$} \\
\hline 10 & $4.53 \mathrm{E}-03$ & E & $1.08 \mathrm{E}-02$ & - & $3.74 \mathrm{E}-03$ & - & $1.15 \mathrm{E}-02$ & - \\
\hline 20 & \begin{tabular}{|l|}
$4.96 \mathrm{E}-04$ \\
\end{tabular} & 3.19 & $1.98 \mathrm{E}-03$ & 2.45 & $3.02 \mathrm{E}-04$ & 3.63 & $1.07 \mathrm{E}-03$ & 3.42 \\
\hline 40 & \begin{tabular}{|l}
$8.80 \mathrm{E}-06$ \\
\end{tabular} & 5.82 & $2.51 \mathrm{E}-05$ & 6.30 & $4.03 \mathrm{E}-06$ & 6.23 & $2.74 \mathrm{E}-05$ & 5.29 \\
\hline 80 & \begin{tabular}{|l}
$8.97 \mathrm{E}-07$ \\
\end{tabular} & 3.29 & $2.91 \mathrm{E}-06$ & 3.11 & $1.74 \mathrm{E}-09$ & 11.18 & $1.32 \mathrm{E}-08$ & 11.02 \\
\hline 160 & $1.12 \mathrm{E}-07$ & 3.00 & $3.64 \mathrm{E}-07$ & 3.00 & $5.09 \mathrm{E}-11$ & 5.09 & $8.75 \mathrm{E}-11$ & $\overline{7.23}$ \\
\hline \multicolumn{9}{|c|}{$\overline{P^{3}}$} \\
\hline 10 & $2.87 \mathrm{E}-03$ & - & $1.24 \mathrm{E}-02$ & - & $7.76 \mathrm{E}-04$ & - & $1.81 \mathrm{E}-03$ & \\
\hline 20 & \begin{tabular}{|l|}
$1.97 \mathrm{E}-04$ \\
\end{tabular} & 3.87 & $1.03 \mathrm{E}-03$ & 3.60 & $6.91 \mathrm{E}-06$ & 6.81 & $2.92 \mathrm{E}-05$ & 5.95 \\
\hline$\overline{40}$ & \begin{tabular}{|c|}
$1.36 \mathrm{E}-06$ \\
\end{tabular} & 7.18 & $7.21 \mathrm{E}-06$ & 7.15 & $3.51 \mathrm{E}-08$ & 7.62 & $1.88 \mathrm{E}-07$ & 7.27 \\
\hline 80 & \begin{tabular}{|c|}
$3.03 \mathrm{E}-09$ \\
\end{tabular} & 8.81 & $1.01 \mathrm{E}-08$ & 9.47 & $2.18 \mathrm{E}-11$ & 10.65 & $6.89 \mathrm{E}-11$ & 11.42 \\
\hline \multicolumn{9}{|c|}{$\overline{P^{4}}$} \\
\hline 10 & \begin{tabular}{|l|}
$1.93 \mathrm{E}-03$ \\
\end{tabular} & - & $6.32 \mathrm{E}-03$ & E & $1.36 \mathrm{E}-03$ & - & $2.91 \mathrm{E}-03$ & - \\
\hline 20 & \begin{tabular}{|c|}
$9.79 \mathrm{E}-05$ \\
\end{tabular} & 4.30 & $5.42 \mathrm{E}-04$ & 3.54 & $1.15 \mathrm{E}-07$ & 13.53 & $8.37 \mathrm{E}-07$ & 11.76 \\
\hline 40 & \begin{tabular}{|c|}
$5.86 \mathrm{E}-07$ \\
\end{tabular} & 7.39 & $4.70 \mathrm{E}-06$ & 6.85 & $3.46 \mathrm{E}-11$ & 11.70 & $2.11 \mathrm{E}-10$ & 11.96 \\
\hline
\end{tabular}

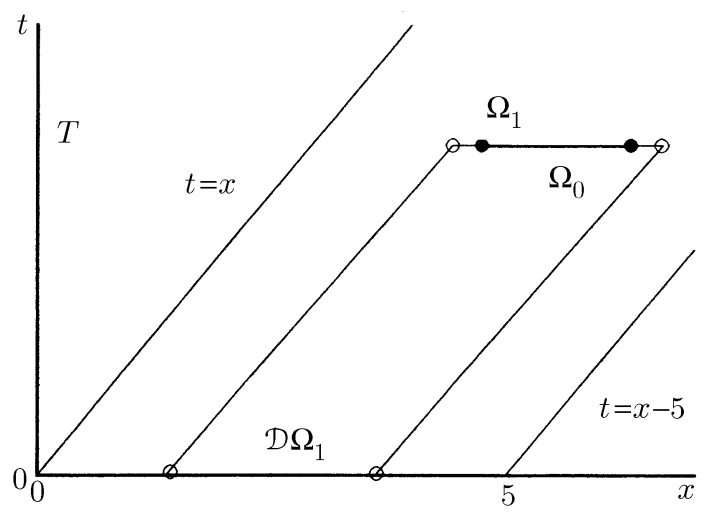

FiguRE 5.4. Sketch of the domain of smoothness $\Omega_{0}$ of the exact solution, the domain $\Omega_{1}$ and its corresponding domain of dependence $\mathcal{D} \Omega_{1}$. The pattern between the discontinuities $t=x$ and $t=x-5$ should be repeated periodically.

the $L^{2}$-errors and the $L^{\infty}$-errors are still at least $(2 k+1)$-th order accurate for $P^{k}$-elements after post-processing. This indicates that it is reasonable to expect 
TABLE 5.4. Example 5.4, Burgers' equation with smooth solution.

\begin{tabular}{|c|c|c|c|c|c|c|c|c|}
\hline & \multicolumn{4}{|c|}{ Before post-processing } & \multicolumn{4}{|c|}{ After post-processing } \\
\hline mesh & $L^{2}$-error & order & $L^{\infty}$-error & order & $L^{2}$-error & order & $L^{\infty}$-error & order \\
\hline \multicolumn{9}{|c|}{$P^{1}$} \\
\hline 10 & $1.95 \mathrm{E}-02$ & E & $8.87 \mathrm{E}-02$ & E & $1.37 \mathrm{E}-02$ & E & $3.99 \mathrm{E}-02$ & \\
\hline 20 & \begin{tabular}{|l}
$5.31 \mathrm{E}-03$ \\
\end{tabular} & 1.88 & $2.77 \mathrm{E}-02$ & 1.68 & $1.63 \mathrm{E}-03$ & 3.07 & $6.47 \mathrm{E}-03$ & 2.63 \\
\hline 40 & $1.33 \mathrm{E}-03$ & 2.00 & $7.55 \mathrm{E}-03$ & 1.87 & $1.28 \mathrm{E}-04$ & 3.67 & $5.55 \mathrm{E}-04$ & 3.54 \\
\hline 80 & $3.33 \mathrm{E}-04$ & 1.99 & $1.95 \mathrm{E}-03$ & 1.95 & $1.03 \mathrm{E}-05$ & 3.63 & $4.17 \mathrm{E}-05$ & 3.73 \\
\hline 160 & \begin{tabular}{|l|}
$8.37 \mathrm{E}-05$ \\
\end{tabular} & 1.99 & $4.99 \mathrm{E}-04$ & 1.97 & $1.12 \mathrm{E}-06$ & 3.20 & $4.21 \mathrm{E}-06$ & 3.31 \\
\hline 320 & \begin{tabular}{|l|}
$2.10 \mathrm{E}-05$ \\
\end{tabular} & 2.00 & $1.26 \mathrm{E}-04$ & 1.98 & $1.42 \mathrm{E}-07$ & 2.98 & $5.69 \mathrm{E}-07$ & 2.89 \\
\hline \multicolumn{9}{|c|}{$P^{2}$} \\
\hline 10 & $3.46 \mathrm{E}-03$ & - & $1.93 \mathrm{E}-02$ & ב & $1.12 \mathrm{E}-02$ & - & $3.37 \mathrm{E}-02$ & - \\
\hline 20 & $4.81 \mathrm{E}-04$ & 2.85 & $3.57 \mathrm{E}-03$ & 2.43 & $9.25 \mathrm{E}-04$ & 3.59 & $3.47 \mathrm{E}-03$ & 3.28 \\
\hline 40 & \begin{tabular}{|l}
$8.00 \mathrm{E}-05$ \\
\end{tabular} & 2.59 & $6.22 \mathrm{E}-04$ & 2.52 & $3.63 \mathrm{E}-05$ & 4.67 & $1.58 \mathrm{E}-04$ & 4.46 \\
\hline 80 & $1.30 \mathrm{E}-05$ & 2.62 & 1.2 & 2.37 & $8.43 \mathrm{E}-07$ & 5.43 & E-06 & 5.33 \\
\hline 160 & \begin{tabular}{|l}
$2.04 \mathrm{E}-06$ \\
\end{tabular} & 2.67 & 1.9 & 2.61 & $1.67 \mathrm{E}-08$ & 5.66 & $\begin{array}{c}\mathrm{i}-08 \\
-08\end{array}$ & 5.53 \\
\hline 320 & \begin{tabular}{|l}
$3.06 \mathrm{E}-07$ \\
\end{tabular} & 2.73 & $3.02 \mathrm{E}-06$ & 2.71 & $3.60 \mathrm{E}-10$ & 5.53 & $1.85 \mathrm{E}-09$ & 5.52 \\
\hline \multicolumn{9}{|c|}{$P^{3}$} \\
\hline 10 & $4.33 \mathrm{E}-04$ & - & $2.24 \mathrm{E}-03$ & - & $1.12 \mathrm{E}-02$ & - & $3.35 \mathrm{E}-02$ & \\
\hline 20 & $4.16 \mathrm{E}-05$ & 3.38 & 2.00 & 3.48 & $8.08 \mathrm{E}-04$ & 3.80 & $\mathrm{E}-03$ & 3.46 \\
\hline 40 & \begin{tabular}{|l|}
$2.43 \mathrm{E}-06$ \\
\end{tabular} & 4.10 & $1.74 \mathrm{E}-05$ & 3.53 & $2.06 \mathrm{E}-05$ & 5.30 & $9.42 \mathrm{E}-05$ & $\overline{5.01}$ \\
\hline 80 & \begin{tabular}{|c|}
$1.46 \mathrm{E}-07$ \\
\end{tabular} & 4.06 & $1.04 \mathrm{E}-06$ & 4.07 & $1.96 \mathrm{E}-07$ & 6.72 & $1.01 \mathrm{E}-06$ & 6.54 \\
\hline 160 & $1.03 \mathrm{E}-08$ & 3.82 & $6.72 \mathrm{E}-08$ & 3.95 & $1.10 \mathrm{E}-09$ & 7.47 & $5.94 \mathrm{E}-09$ & 7.41 \\
\hline \multicolumn{9}{|c|}{$P^{4}$} \\
\hline 10 & $1.75 \mathrm{E}-04$ & $\bar{E}$ & $8.25 \mathrm{E}-04$ & 二 & $1.15 \mathrm{E}-02$ & $=$ & $3.36 \mathrm{E}-02$ & \\
\hline 20 & $4.19 \mathrm{E}-06$ & 5.39 & $2.45 \mathrm{E}-05$ & 5.07 & $7.63 \mathrm{E}-04$ & 3.91 & $2.82 \mathrm{E}-03$ & 3.58 \\
\hline 40 & \begin{tabular}{|l}
$1.70 \mathrm{E}-07$ \\
\end{tabular} & 4.62 & $1.04 \mathrm{E}-06$ & 4.55 & $1.48 \mathrm{E}-05$ & 5.69 & $6.82 \mathrm{E}-05$ & 5.37 \\
\hline$\overline{50}$ & \begin{tabular}{|c|}
$6.45 \mathrm{E}-08$ \\
\end{tabular} & 4.36 & $4.40 \mathrm{E}-07$ & 3.87 & $3.09 \mathrm{E}-06$ & 7.03 & $1.52 \mathrm{E}-05$ & 6.74 \\
\hline
\end{tabular}

that a similar result with a domain $\mathcal{D} \Omega_{1}$ excluding the discontinuity should hold, see Figure 5.4

In Figure 5.3 we plot the errors, in absolute value and in logarithmic scale, of the numerical solution before and after post-processing for $P^{2}$, with 10, 20, 40, 80 and 160 elements. We can clearly see that the post-processed errors are less oscillatory and much smaller in magnitude away from the discontinuity.

Example 5.4. A scalar nonlinear Burgers' equation with continuous and discontinuous solutions on the domain $I=[0,2 \pi)$ :

$$
u_{t}+\left(\frac{1}{2} u^{2}\right)_{x}=0, \quad \text { in } I \times(0, T), \quad u(x, 0)=\frac{1}{2}+\sin (x), \quad x \in I,
$$

with periodic boundary conditions. The errors at $T=0.5$, when the solution is still smooth, are given in Table 5.4. It seems that in general, post-processed errors are still smaller, although the asymptotic orders seem to show up later than for the linear case, as the mesh is refined. We remark that the theory in this paper does not cover this nonlinear problem.

In Figure 5.5 we plot the errors of the numerical solution before and after postprocessing for $P^{2}$ and $P^{3}$ with 20 elements. From Table 5.4 we can see that in both situations the errors after post-processing are actually larger than the errors before 

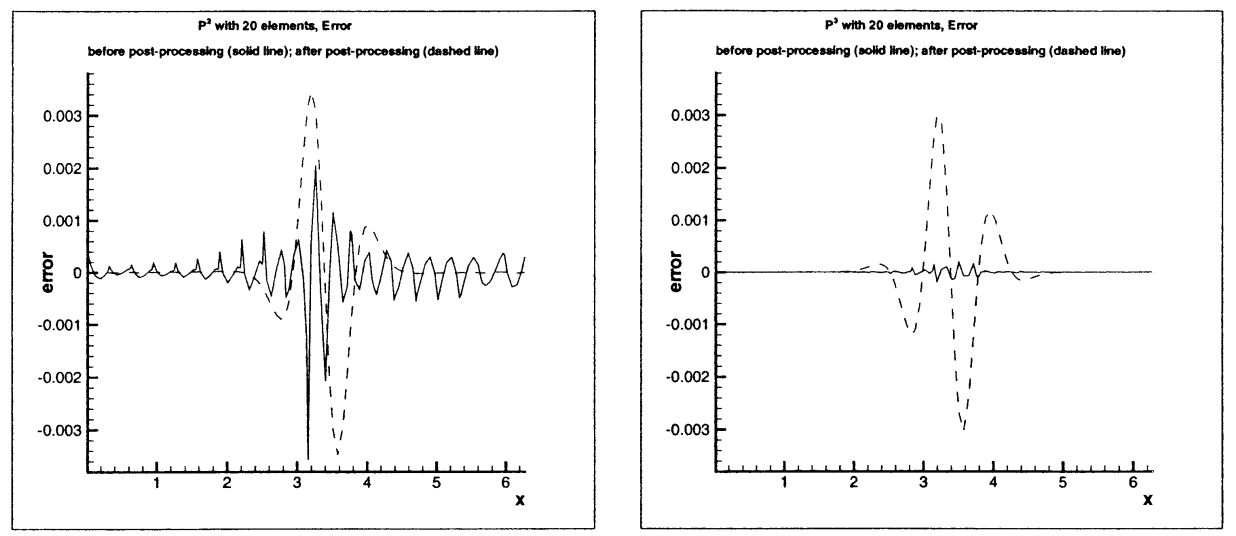

Figure 5.5. The errors before and after post-processing for the smooth solution of Burgers equation and 20 elements: $P^{2}$ (left) and $P^{3}$ (right).
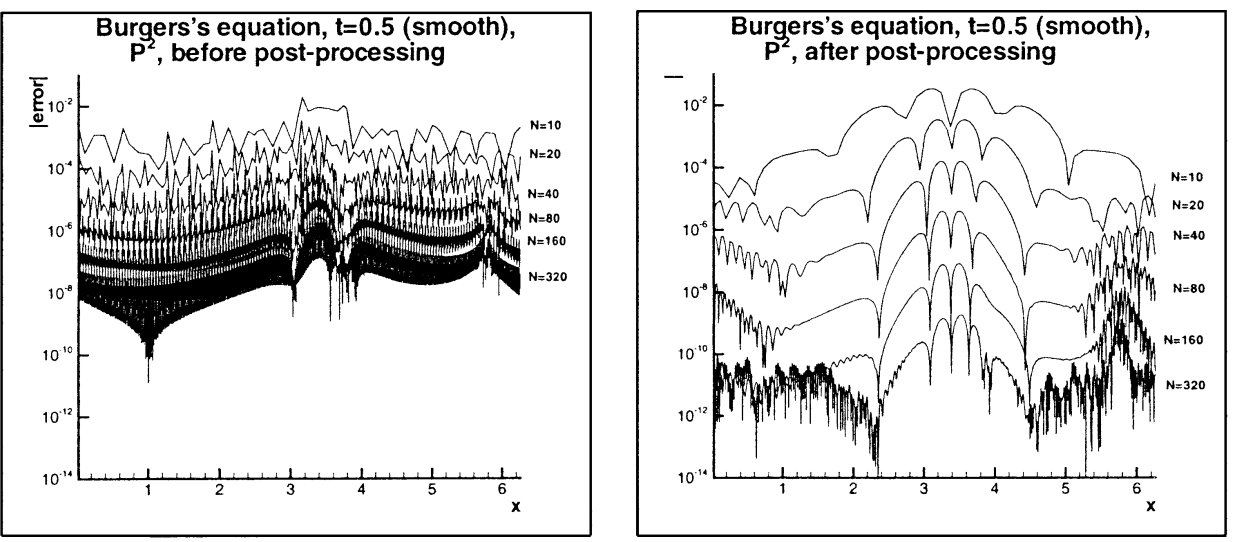

Figure 5.6. The errors in absolute value and in logarithmic scale, for $P^{2}$ with $\mathrm{N}=20,40,40,80,160$ and 320 elements. Smooth solution of Burgers equation. Before post-processing (left) and after post-processing (right).

post-processing. Note in Figure 5.5 that near the middle region, the oscillations in the errors are not "uniform", apparently due to nonlinear effects, hence the postprocessing actually gives larger errors. Fortunately, for a larger number of elements, the post-processing begins to be effective and the errors after post-processing do become smaller; see Table 5.4 and Figure 5.6

In Figure 5.6 we plot the errors, in absolute value and in logarithmic scale, of the numerical solution before and after post-processing for $P^{2}$, with $10,20,40$, 80,160 and 320 elements. We can clearly see that the post-processed errors are less oscillatory and much smaller in magnitude, especially for a large number of elements. However, notice that due to nonlinear effects not all oscillations in the 
TABLE 5.5. Example 5.4, Burgers' equation with discontinuous solution.

\begin{tabular}{|c|c|c|c|c|c|c|c|c|}
\hline & \multicolumn{4}{|c|}{ Before post-processing } & \multicolumn{4}{|c|}{ After post-processing } \\
\hline mesh & $L^{2}$-error & order & $L^{\infty}$-error & order & $L^{2}$-error & order & $L^{\infty}$-error & order \\
\hline \multicolumn{9}{|c|}{$P^{1}$} \\
\hline 10 & $8.70 \mathrm{E}-03$ & E & $3.56 \mathrm{E}-02$ & $\overline{-}$ & $6.79 \mathrm{E}-03$ & E & $1.99 \mathrm{E}-02$ & - \\
\hline 20 & $3.05 \mathrm{E}-04$ & 4.83 & $1.47 \mathrm{E}-03$ & 4.60 & $2.23 \mathrm{E}-04$ & 4.93 & $8.61 \mathrm{E}-04$ & 4.53 \\
\hline 40 & $1.70 \mathrm{E}-05$ & 4.16 & $8.14 \mathrm{E}-05$ & 4.18 & $1.09 \mathrm{E}-05$ & 4.36 & $2.25 \mathrm{E}-05$ & 5.26 \\
\hline 80 & $3.71 \mathrm{E}-06$ & 2.20 & $2.07 \mathrm{E}-05$ & 1.97 & $1.37 \mathrm{E}-06$ & 2.99 & $2.86 \mathrm{E}-06$ & 2.97 \\
\hline 160 & $8.65 \mathrm{E}-07$ & 2.10 & $4.67 \mathrm{E}-06$ & 2.15 & $1.63 \mathrm{E}-07$ & 3.07 & $3.43 \mathrm{E}-07$ & 3.06 \\
\hline 320 & $2.17 \mathrm{E}-07$ & 2.00 & $1.19 \mathrm{E}-06$ & 1.97 & $2.05 \mathrm{E}-08$ & 3.00 & $4.31 \mathrm{E}-08$ & 2.99 \\
\hline \multicolumn{9}{|c|}{$P^{2}$} \\
\hline 10 & $6.26 \mathrm{E}-03$ & $=$ & $3.29 \mathrm{E}-02$ & - & $1.57 \mathrm{E}-03$ & $=$ & $7.05 \mathrm{E}-03$ & \\
\hline 20 & $2.77 \mathrm{E}-04$ & 4.50 & $1.44 \mathrm{E}-03$ & 4.52 & $5.47 \mathrm{E}-05$ & 4.84 & $1.52 \mathrm{E}-04$ & 5.54 \\
\hline 40 & $2.03 \mathrm{E}-05$ & 3.77 & $1.68 \mathrm{E}-04$ & 3.10 & $6.88 \mathrm{E}-06$ & 2.99 & $2.62 \mathrm{E}-05$ & 2.53 \\
\hline 80 & $2.30 \mathrm{E}-06$ & 3.14 & $2.17 \mathrm{E}-05$ & 2.95 & $8.39 \mathrm{E}-07$ & 3.03 & $4.61 \mathrm{E}-06$ & 2.51 \\
\hline 160 & $4.23 \mathrm{E}-07$ & 2.44 & $4.75 \mathrm{E}-06$ & 2.19 & $1.16 \mathrm{E}-07$ & 2.86 & $7.95 \mathrm{E}-07$ & 2.54 \\
\hline 320 & $6.12 \mathrm{E}-08$ & 2.79 & $7.77 \mathrm{E}-07$ & 2.61 & $1.41 \mathrm{E}-08$ & $\overline{3.04}$ & $1.29 \mathrm{E}-07$ & 2.62 \\
\hline
\end{tabular}
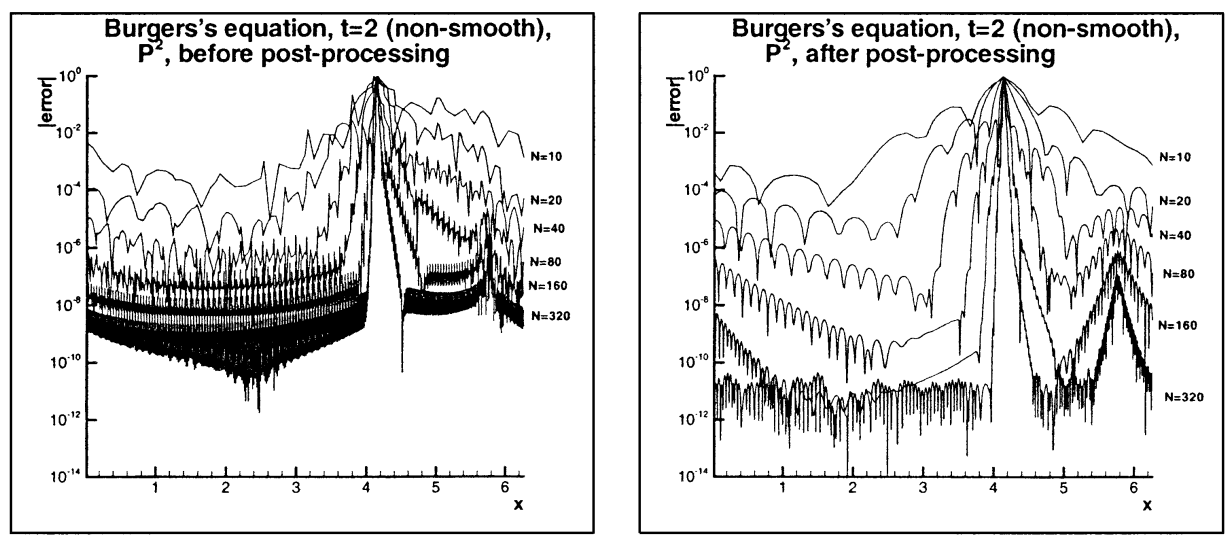

FiguRE 5.7. The errors in absolute value and in logarithmic scale, for $P^{2}$ with $\mathrm{N}=10,20,40,40,80,160$ and 320 elements. Discontinuous solution of Burgers equation. Before post-processing (left) and after post-processing (right).

errors have been removed by the post-processing, especially for a large number of elements.

Next, we compute the solution at $T=2$, that is, after the shock has developed. We measure the errors on the smooth region $0.5 \pi$ away from the discontinuity and show the results in Table 5.5. The codes ran stably only for $P^{1}$ and $P^{2}$ hence only these two cases are shown.

In order to stabilize the algorithm in the presence of shocks, we apply a TVB (total variation bounded) limiter with $M=3$, see [8]. This limiter has no effect on the numerical solution at $T=0.5$ when the solution is still smooth, but allows the scheme to run stably for $P^{3}$ and $P^{4}$ after the shock develops. We again measure 
TABLE 5.6. Example 5.4, Burgers' equation with discontinuous solution. TVB limiters.

\begin{tabular}{|c|c|c|c|c|c|c|c|c|}
\hline & \multicolumn{4}{|c|}{ Before post-processing } & \multicolumn{4}{|c|}{ After post-processing } \\
\hline mesh & $L^{2}$-error & order & $L^{\infty}$-error & order & $L^{2}$-error & order & $L^{\infty}$-error & order \\
\hline \multicolumn{9}{|c|}{$P^{1}$} \\
\hline 10 & $1.26 \mathrm{E}-03$ & - & $4.44 \mathrm{E}-03$ & - & $1.02 \mathrm{E}-03$ & - & $2.28 \mathrm{E}-03$ & - \\
\hline 20 & \begin{tabular}{|c|}
$1.16 \mathrm{E}-04$ \\
\end{tabular} & 3.44 & $4.38 \mathrm{E}-04$ & 3.34 & $1.01 \mathrm{E}-04$ & 3.33 & $1.94 \mathrm{E}-04$ & 3.55 \\
\hline 40 & \begin{tabular}{|l|}
$1.72 \mathrm{E}-05$ \\
\end{tabular} & 2.76 & $8.33 \mathrm{E}-05$ & 2.40 & $1.09 \mathrm{E}-05$ & 3.22 & $2.26 \mathrm{E}-05$ & 3.11 \\
\hline 80 & \begin{tabular}{|c|}
$3.72 \mathrm{E}-06$ \\
\end{tabular} & 2.20 & $2.08 \mathrm{E}-05$ & 2.00 & $1.37 \mathrm{E}-06$ & 2.99 & $2.87 \mathrm{E}-06$ & 2.98 \\
\hline 160 & \begin{tabular}{|l|}
$8.73 \mathrm{E}-07$ \\
\end{tabular} & 2.09 & $4.75 \mathrm{E}-06$ & 2.13 & $1.63 \mathrm{E}-07$ & 3.07 & $3.44 \mathrm{E}-07$ & 3.06 \\
\hline 320 & \begin{tabular}{|l|}
$2.17 \mathrm{E}-07$ \\
\end{tabular} & 2.01 & $1.19 \mathrm{E}-06$ & 2.00 & $2.05 \mathrm{E}-08$ & 3.00 & $4.32 \mathrm{E}-08$ & 2.99 \\
\hline \multicolumn{9}{|c|}{$\overline{P^{2}}$} \\
\hline 10 & $1.03 \mathrm{E}-02$ & - & $5.83 \mathrm{E}-02$ & $=$ & $1.99 \mathrm{E}-03$ & $=$ & $7.66 \mathrm{E}-03$ & 二 \\
\hline 20 & \begin{tabular}{|l|}
$4.22 \mathrm{E}-04$ \\
\end{tabular} & 4.60 & 3.3 & 4.12 & $5.32 \mathrm{E}-05$ & 5.22 & -04 & 5.68 \\
\hline 40 & \begin{tabular}{|l|}
$2.23 \mathrm{E}-05$ \\
\end{tabular} & 4.24 & 1.9 & 4.08 & $6.87 \mathrm{E}-06$ & 2.95 & -05 & 2.53 \\
\hline 80 & \begin{tabular}{|c|}
$2.29 \mathrm{E}-06$ \\
\end{tabular} & 3.28 & 2.1 & 3.20 & $8.39 \mathrm{E}-07$ & 3.03 & -06 & 2.50 \\
\hline 160 & \begin{tabular}{|l}
$4.21 \mathrm{E}-07$ \\
\end{tabular} & 2.44 & 4.7 & 2.19 & $1.16 \mathrm{E}-07$ & 2.86 & $7.95 \mathrm{E}-07$ & 2.54 \\
\hline 320 & \begin{tabular}{|c|}
$6.10 \mathrm{E}-08$ \\
\end{tabular} & 2.79 & $7.75 \mathrm{E}-07$ & 2.61 & $1.41 \mathrm{E}-08$ & 3.04 & $1.29 \mathrm{E}-07$ & 2.62 \\
\hline \multicolumn{9}{|c|}{$P^{3}$} \\
\hline 10 & \begin{tabular}{|c|}
$9.98 \mathrm{E}-04$ \\
\end{tabular} & $=$ & 6.6 & - & $3.45 \mathrm{E}-03$ & ב- & $1.35 \mathrm{E}-02$ & \\
\hline 20 & \begin{tabular}{|c|}
$1.47 \mathrm{E}-04$ \\
\end{tabular} & 2.76 & $1.38 \mathrm{E}-03$ & 2.20 & $9.35 \mathrm{E}-06$ & 8.52 & $5.18 \mathrm{E}-05$ & 8.03 \\
\hline 40 & \begin{tabular}{|l|}
$4.92 \mathrm{E}-07$ \\
\end{tabular} & 8.22 & $5.43 \mathrm{E}-06$ & 7.99 & $2.92 \mathrm{E}-08$ & 8.32 & $2.08 \mathrm{E}-07$ & 7.96 \\
\hline 80 & \begin{tabular}{|l|}
$8.58 \mathrm{E}-10$ \\
\end{tabular} & 9.16 & $1.43 \mathrm{E}-08$ & 8.57 & $3.71 \mathrm{E}-10$ & 6.30 & $8.73 \mathrm{E}-10$ & 7.90 \\
\hline \multicolumn{9}{|c|}{$P^{4}$} \\
\hline 10 & \begin{tabular}{|l|}
$3.86 \mathrm{E}-01$ \\
\end{tabular} & - & $9.90 \mathrm{E}-01$ & - & $2.28 \mathrm{E}-01$ & - & $3.78 \mathrm{E}-01$ & \\
\hline 20 & \begin{tabular}{|c|}
$1.08 \mathrm{E}-01$ \\
\end{tabular} & 1.84 & 2.1 & 2.20 & $5.28 \mathrm{E}-02$ & 2.11 & $1.34 \mathrm{E}-01$ & 1.50 \\
\hline 40 & \begin{tabular}{|c|}
$1.89 \mathrm{E}-03$ \\
\end{tabular} & 5.83 & & 3.26 & $3.88 \mathrm{E}-04$ & 7.09 & Q-03 & 5.07 \\
\hline 80 & \begin{tabular}{|c|}
$8.08 \mathrm{E}-08$ \\
\end{tabular} & 14.52 & $6.11 \mathrm{E}-07$ & 15.17 & $1.46 \mathrm{E}-08$ & 14.70 & $7.42 \mathrm{E}-08$ & 15.71 \\
\hline
\end{tabular}

the errors on the smooth region $0.5 \pi$ away from the discontinuity and show the result in Table 5.6 .

In Figure 5.7 we plot the errors, in absolute value and in logarithmic scale, of the numerical solution before and after post-processing for $P^{2}$ with a TVB limiter, at $t=2$, with $10,20,40,80,160$ and 320 elements. We can clearly see that the post-processed errors are less oscillatory and much smaller in magnitude, especially for a large number of elements, away from the discontinuity. Again, notice that not all oscillations in the errors have been removed by the post-processing, especially for a large number of elements, due to nonlinear effects.

Example 5.5. A linear system with smooth solution in the domain $I=[0,2 \pi)$ :

$$
\left\{\begin{array}{l}
u_{t}+v_{x}=0 \\
v_{t}+u_{x}=0
\end{array} \quad \text { in } I \times(0, T), \quad\left\{\begin{array}{l}
u(x, 0)=\sin (x) \\
v(x, 0)=0
\end{array} \quad x \in I,\right.\right.
$$

with periodic boundary conditions. The errors are computed at $T=12.5$ which is about two periods in time.

In Table [5.7, we show the results for this problem. The errors are the combined ones of $u$ and $v$. We can clearly see that both $L^{2}$ - and $L^{\infty}$-errors for $P^{k}$-elements 
TABLE 5.7. Example 5.5, linear system with smooth solution.

\begin{tabular}{|c|c|c|c|c|c|c|c|c|}
\hline & \multicolumn{4}{|c|}{ Before post-processing } & \multicolumn{4}{|c|}{ After post-processing } \\
\hline mesh & $L^{2}$-error & order & $L^{\infty}$-error & order & $L^{2}$-error & order & $L^{\infty}$-error & order \\
\hline \multicolumn{9}{|c|}{$\overline{P^{1}}$} \\
\hline 10 & $2.33 \mathrm{E}-02$ & E & $5.20 \mathrm{E}-02$ & - & $2.13 \mathrm{E}-02$ & 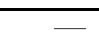 & $4.16 \mathrm{E}-02$ & - \\
\hline 20 & \begin{tabular}{|c|}
$3.98 \mathrm{E}-03$ \\
\end{tabular} & 2.55 & $8.55 \mathrm{E}-03$ & 2.60 & $2.72 \mathrm{E}-03$ & 2.97 & $5.36 \mathrm{E}-03$ & 2.96 \\
\hline 40 & $8.20 \mathrm{E}-04$ & 2.28 & $1.89 \mathrm{E}-03$ & 2.18 & $3.39 \mathrm{E}-04$ & 3.00 & $6.74 \mathrm{E}-04$ & 2.99 \\
\hline 80 & \begin{tabular}{|l|}
$1.92 \mathrm{E}-04$ \\
\end{tabular} & 2.09 & $4.77 \mathrm{E}-04$ & 1.98 & $4.23 \mathrm{E}-05$ & 3.00 & $8.43 \mathrm{E}-05$ & 3.00 \\
\hline 160 & $4.72 \mathrm{E}-05$ & 2.03 & $1.20 \mathrm{E}-04$ & 2.00 & $5.28 \mathrm{E}-06$ & 3.00 & $1.05 \mathrm{E}-05$ & 3.00 \\
\hline 320 & \begin{tabular}{|l}
$1.17 \mathrm{E}-05$ \\
\end{tabular} & 2.01 & $2.99 \mathrm{E}-05$ & 2.00 & $6.59 \mathrm{E}-07$ & 3.00 & $1.31 \mathrm{E}-06$ & 3.00 \\
\hline \multicolumn{9}{|c|}{$P^{2}$} \\
\hline 10 & $6.10 \mathrm{E}-04$ & $=$ & $1.67 \mathrm{E}-03$ & $=$ & $1.78 \mathrm{E}-04$ & - & $3.53 \mathrm{E}-04$ & \\
\hline 20 & \begin{tabular}{|l|}
$7.57 \mathrm{E}-05$ \\
\end{tabular} & 3.01 & $2.08 \mathrm{E}-04$ & 3.00 & $4.22 \mathrm{E}-06$ & 5.40 & $8.42 \mathrm{E}-06$ & 5.39 \\
\hline$\overline{40}$ & \begin{tabular}{|l}
$9.46 \mathrm{E}-06$ \\
\end{tabular} & 3.00 & $2.60 \mathrm{E}-05$ & 3.00 & $1.09 \mathrm{E}-07$ & 5.28 & $2.17 \mathrm{E}-07$ & 5.28 \\
\hline 80 & $1.18 \mathrm{E}-06$ & 3.00 & $3.24 \mathrm{E}-06$ & 3.00 & $3.11 \mathrm{E}-09$ & 5.13 & $6.20 \mathrm{E}-09$ & $\overline{5.13}$ \\
\hline 160 & \begin{tabular}{|l}
$1.48 \mathrm{E}-07$ \\
\end{tabular} & 3.00 & $4.06 \mathrm{E}-07$ & 3.00 & $8.95 \mathrm{E}-11$ & 5.12 & $1.77 \mathrm{E}-10$ & 5.13 \\
\hline \multicolumn{9}{|c|}{$\overline{P^{3}}$} \\
\hline 10 & $2.33 \mathrm{E}-05$ & E- & $5.73 \mathrm{E}-05$ & - & $1.16 \mathrm{E}-05$ & - & $2.30 \mathrm{E}-05$ & - \\
\hline 20 & \begin{tabular}{|l}
$1.46 \mathrm{E}-06$ \\
\end{tabular} & 4.00 & $3.61 \mathrm{E}-06$ & 3.99 & $5.00 \mathrm{E}-08$ & 7.85 & $9.98 \mathrm{E}-08$ & 7.85 \\
\hline 40 & \begin{tabular}{|l|}
$9.13 \mathrm{E}-08$ \\
\end{tabular} & 4.00 & $2.27 \mathrm{E}-07$ & 3.99 & $2.13 \mathrm{E}-10$ & 7.88 & $4.25 \mathrm{E}-10$ & 7.88 \\
\hline 50 & \begin{tabular}{|c|}
$3.74 \mathrm{E}-08$ \\
\end{tabular} & 4.00 & $9.29 \mathrm{E}-08$ & 4.01 & $3.94 \mathrm{E}-11$ & 7.56 & $7.84 \mathrm{E}-11$ & 7.57 \\
\hline \multicolumn{9}{|c|}{$P^{4}$} \\
\hline 10 & \begin{tabular}{|l}
$7.24 \mathrm{E}-07$ \\
\end{tabular} & E & $1.37 \mathrm{E}-06$ & $=$ & $1.40 \mathrm{E}-06$ & E & $2.79 \mathrm{E}-06$ & \\
\hline 20 & \begin{tabular}{|l|}
$2.27 \mathrm{E}-08$ \\
\end{tabular} & 5.00 & $4.33 \mathrm{E}-08$ & 4.99 & $1.56 \mathrm{E}-09$ & 9.82 & $3.11 \mathrm{E}-09$ & 9.81 \\
\hline 30 & \begin{tabular}{|c|}
$2.99 \mathrm{E}-09$ \\
\end{tabular} & 5.00 & $5.72 \mathrm{E}-09$ & 4.99 & $3.06 \mathrm{E}-11$ & 9.69 & $6.16 \mathrm{E}-11$ & 9.67 \\
\hline
\end{tabular}

are $(k+1)$-th order before post-processing and at least $(2 k+1)$-th order after postprocessing. In fact, the errors are very similar to the scalar case in Example 5.1. This is consistent with our theoretical results.

Notice that this example and the next one with a discontinuous solution for linear systems indicate that the method is very suitable for long time simulation of linear systems as the post-processing needs to be performed only at the final time. Examples include aeroacoustic problems when linear Euler equations must be solved for a long time to propagate the pressure waves.

Example 5.6. The same linear system (5.4) with the same initial condition, except that now $0 \leq x<5$ and the boundary condition is 5 -periodic. The solution now has a discontinuity at $x=0$ (or $x=5$ ) and this discontinuity moves in time with the characteristic speeds \pm 1 . We compute the errors at $t=12.5$, after 2.5 periods in time. The two discontinuities at this time are both located at $x=2.5$. The errors shown in Table 5.8 are calculated within the "smooth region" that lies a distance 1.5 away from the discontinuities, namely excluding the interval $1<x<4$.

The theory in this paper would only guarantee $(k+1)$-th order accuracy for $P^{k}$ elements after post-processing, since when we take $\mathcal{D} \Omega_{1}=I$, the initial condition has a discontinuity in this set. However, Table 5.8 shows that both the $L^{2}$-errors and the $L^{\infty}$-errors are still at least $(2 k+1)$-th order accurate for $P^{k}$-elements after post-processing. 
TABLE 5.8. Example 5.6, linear system with discontinuous solution.

\begin{tabular}{|c|c|c|c|c|c|c|c|c|}
\hline & \multicolumn{4}{|c|}{ Before post-processing } & \multicolumn{4}{|c|}{ After post-processing } \\
\hline mesh & $L^{2}$-error & order & $L^{\infty}$-error & order & $L^{2}$-error & order & $L^{\infty}$-error & order \\
\hline \multicolumn{9}{|c|}{$P^{1}$} \\
\hline 10 & $1.49 \mathrm{E}-02$ & $=$ & $4.00 \mathrm{E}-02$ & $=$ & $1.29 \mathrm{E}-02$ & $=$ & $4.27 \mathrm{E}-02$ & - \\
\hline 20 & $3.19 \mathrm{E}-03$ & 2.22 & $9.35 \mathrm{E}-03$ & 2.10 & $2.79 \mathrm{E}-03$ & 2.21 & $7.69 \mathrm{E}-03$ & 2.47 \\
\hline 40 & $4.76 \mathrm{E}-04$ & 2.74 & $1.37 \mathrm{E}-03$ & 2.78 & $1.85 \mathrm{E}-04$ & 3.91 & $4.82 \mathrm{E}-04$ & 3.99 \\
\hline 80 & $1.13 \mathrm{E}-04$ & 2.08 & $3.04 \mathrm{E}-04$ & 2.17 & $1.99 \mathrm{E}-05$ & 3.22 & $4.28 \mathrm{E}-05$ & 3.49 \\
\hline 160 & $2.78 \mathrm{E}-05$ & 2.02 & $7.59 \mathrm{E}-05$ & 2.00 & $2.48 \mathrm{E}-06$ & 3.00 & $5.31 \mathrm{E}-06$ & 3.01 \\
\hline 320 & $6.94 \mathrm{E}-06$ & 2.00 & $1.90 \mathrm{E}-05$ & 2.00 & $3.09 \mathrm{E}-07$ & 3.00 & $6.63 \mathrm{E}-07$ & $\overline{3.00}$ \\
\hline \multicolumn{9}{|c|}{$P^{2}$} \\
\hline 10 & $3.41 \mathrm{E}-03$ & E & $6.93 \mathrm{E}-03$ & - & $2.65 \mathrm{E}-03$ & E & $9.29 \mathrm{E}-03$ & - \\
\hline 20 & $3.58 \mathrm{E}-04$ & 3.25 & $1.30 \mathrm{E}-03$ & 2.42 & $2.22 \mathrm{E}-04$ & 3.58 & $6.40 \mathrm{E}-04$ & 3.86 \\
\hline 40 & $6.30 \mathrm{E}-06$ & 5.83 & $2.42 \mathrm{E}-05$ & 5.74 & $2.85 \mathrm{E}-06$ & 6.28 & $1.39 \mathrm{E}-05$ & $\overline{5.52}$ \\
\hline 80 & $6.33 \mathrm{E}-07$ & 3.32 & $1.64 \mathrm{E}-06$ & 3.88 & $1.23 \mathrm{E}-09$ & 11.17 & $7.52 \mathrm{E}-09$ & 10.86 \\
\hline 160 & $7.91 \mathrm{E}-08$ & 3.00 & $2.05 \mathrm{E}-07$ & 3.00 & $3.34 \mathrm{E}-11$ & 5.20 & $5.54 \mathrm{E}-11$ & $\overline{7.08}$ \\
\hline \multicolumn{9}{|c|}{$\overline{P^{3}}$} \\
\hline 10 & $2.03 \mathrm{E}-03$ & - & $6.43 \mathrm{E}-03$ & 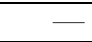 & $5.35 \mathrm{E}-04$ & E & $1.77 \mathrm{E}-03$ & 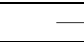 \\
\hline 20 & $1.40 \mathrm{E}-04$ & 3.86 & $5.41 \mathrm{E}-04$ & 3.57 & $4.92 \mathrm{E}-06$ & 6.76 & $2.27 \mathrm{E}-05$ & 6.28 \\
\hline$\overline{40}$ & $9.66 \mathrm{E}-07$ & 7.18 & $3.64 \mathrm{E}-06$ & 7.21 & $2.50 \mathrm{E}-08$ & 7.62 & $9.60 \mathrm{E}-08$ & 7.89 \\
\hline 80 & $2.14 \mathrm{E}-09$ & 8.82 & $6.00 \mathrm{E}-09$ & 9.25 & $1.34 \mathrm{E}-11$ & 10.87 & $4.89 \mathrm{E}-11$ & 10.94 \\
\hline \multicolumn{9}{|c|}{$P^{4}$} \\
\hline$\overline{10}$ & $1.38 \mathrm{E}-03$ & E & $3.26 \mathrm{E}-03$ & 二- & $9.61 \mathrm{E}-04$ & E & $2.90 \mathrm{E}-03$ & \\
\hline 20 & $6.92 \mathrm{E}-05$ & 4.32 & $2.72 \mathrm{E}-04$ & 3.58 & $8.09 \mathrm{E}-08$ & 13.54 & $6.31 \mathrm{E}-07$ & 12.17 \\
\hline 40 & $4.14 \mathrm{E}-07$ & 7.39 & $2.36 \mathrm{E}-06$ & 6.85 & $2.42 \mathrm{E}-11$ & 11.71 & $1.03 \mathrm{E}-10$ & 12.58 \\
\hline
\end{tabular}

In fact, the behavior of the errors is very similar to that observed in the scalar case in Example 5.3. This is not really surprising since our linear system is equivalent to the following two decoupled scalar equations:

$$
\left\{\begin{array}{l}
(u-v)_{t}-(u-v)_{x}=0 \\
(u+v)_{t}+(u+v)_{x}=0
\end{array} \quad \text { in } I \times(0, T), \quad\left\{\begin{array}{l}
(u-v)(x, 0)=\sin (x) \\
(u+v)(x, 0)=\sin (x)
\end{array} \quad x \in I .\right.\right.
$$

As a consequence, the domain of dependence $\mathcal{D} \Omega_{1}$ does not include the discontinuity of the initial condition; see Figure 5.8 (top).

On the other hand, it is interesting to point out that this doubling of the order of accuracy does not take place for the problem

$$
\left\{\begin{array}{l}
u_{t}-u_{x}=v \\
v_{t}+v_{x}=-u
\end{array} \quad \text { in } I \times(0, T), \quad\left\{\begin{array}{l}
u(x, 0)=\sin (x) \\
v(x, 0)=0
\end{array} \quad x \in I,\right.\right.
$$

with periodic boundary conditions, since now the two scalar equations associated with the diagonalization of the system are coupled through zero-order terms; as a consequence, the domain of dependence $\mathcal{D} \Omega_{1}$ always includes the discontinuity of the initial condition; see Figure 5.8 (bottom). This is the example treated in the early work of Majda and Osher and [20] and Majda, McDonough and Osher [19]. Due to this lack of regularity of the initial condition on $\mathcal{D} \Omega_{1}$, post-processing with a kernel of support of order $h$ does not yield any significant improvement; a kernel 

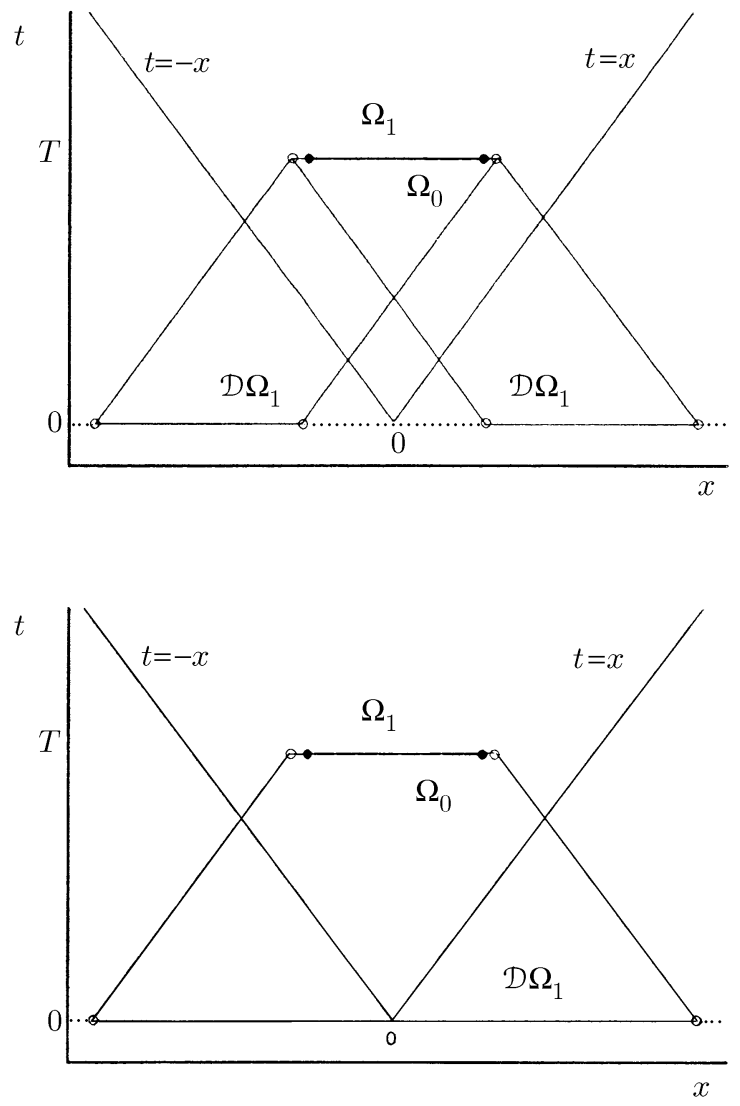

Figure 5.8. The domain of smoothness of $u(T), \Omega_{0}$, the domain $\Omega_{1} \ni \Omega_{0}$ and its corresponding domain of dependence $\mathcal{D} \Omega_{1}$ for the system (5.4) (top) and the system (5.5) (bottom). Note the discontinuity curves $t=|x|$.

of support of order almost one is required, as predicted by our main theorem; see also Mock and Lax [21.

\section{EXTENSIONS AND CONCLUDING REMARKS}

In this paper, we have shown how to enhance the approximation given by a finite element method for linear hyperbolic equations by applying simple post-processing at the very end of the computations. Our theoretical results can be easily extended to the case in which the matrices $A_{j}, j=0, \ldots, d$, are very smooth functions of $(x, t)$. To do that, it is enough to mimic the induction argument presented by Bramble and Schatz in [5].

The role of negative-order error estimates is crucial since it is the analytical tool that captures the ocillatory nature of the error. For these negative-order norms of the error, upper bounds were obtained which depend on a global norm of the initial data. Our numerical results suggest, however, that they should depend only on a local norm of the initial data. In fact, a result of this type was obtained in 1998 
for finite difference schemes by Engquist and Sjögreen [11. To obtain such a result for, say, the discontinuous Galerkin method is a challenging open problem.

Finally, let us end by pointing out that our numerical results seem to indicate that the post-processing has a positive impact on the quality of the approximate solution even if the problem is nonlinear. A theoretical analysis of this case is yet another important open problem.

\section{ACKNowledgments}

The authors are grateful to Paul Castillo for providing the figures in the Introduction and to the reviewer for pointing out the references [4, [15] and 23].

\section{REFERENCES}

1. S. Adjerid, M. Aiffa, and J. E. Flaherty, Computational methods for singularly perturbed systems, Singular Perturbation Concepts of Differential Equations (J. Cronin and R.E. O'Malley, eds.), AMS Proceedings of Symposia in Applied Mathematics, AMS, 1998. MR 2001c:65097

2. S. Adjerid, M. Aiffa, and J.E. Flaherty, High-order finite element methods for singularly perturbed elliptic and parabolic problems, SIAM J. Appl. Math. 55 (1995), 520-543. MR 96d:65182

3. M.Y.T. Apelkrans, Some properties of difference schemes for hyperbolic equations with discontinuities and a new method with almost quadratic convergence, Tech. Report 15A, Uppsala University, Dept. of Computer Science, 1969.

4. L.A. Bales, Some remarks on post-processing and negative norm estimates for approximations to nonsmooth solutions of hyperbolic equations, Comm. Numer. Methods Engrg. 9 (1993), 701-710. CMP 93:17

5. J.H. Bramble and A.H. Schatz, Higher order local accuracy by averaging in the finite element method, Math. Comp. 31 (1977), 94-111. MR 55:4739

6. P. Brenner, V. Thomée, and L.B. Wahlbin, Besov spaces and applications to difference methods for initial value problems, Lecture Notes in Mathematics, vol. 434, Springer Verlag, 1975. MR 57:1106

7. B. Cockburn, Discontinuous Galerkin methods for convection-dominated problems, HighOrder Methods for Computational Physics (T. Barth and H. Deconink, eds.), Lecture Notes in Computational Science and Engineering, vol. 9, Springer Verlag, 1999, pp. 69-224. MR 2000f:76096

8. B. Cockburn and C.-W. Shu, TVB Runge-Kutta local projection discontinuous Galerkin finite element method for scalar conservation laws II: General framework, Math. Comp. 52 (1989), 411-435. MR 90k:65160.

9. - The local discontinuous Galerkin method for time-dependent convection-diffusion systems, SIAM J. Numer. Anal. 35 (1998), 2440-2463. MR 99j:65163

10. J. Douglas, Jr., Superconvergence in the pressure in the simulation of miscible displacement, SIAM J. Numer. Anal. 22 (1985), 962-969. MR 86j:65129

11. B. Engquist and B. Sjögreen, The convergence rate of finite difference schemes in the presence of shocks, SIAM J. Numer. Anal. 35 (1998), 2464-2485. MR 99k:65080

12. R.P. Fedorenko, The application of high-accuracy difference schemes to the numerical solution of hyperbolic equations, Zh. Vychisl. Mat. i Mat. Fiz. 2 (1962), 1122-1128, (in Russian). MR 26:5739

13. D. Gottlieb and E. Tadmor, Recovering pointwise values of discontinuous data within spectral accuracy, Proceedings of U.S.-Israel Workshop, Progress in Scientific Computing, vol. 6, Birkhäuser Boston Inc., 1985, pp. 357-375. MR 90a:65041

14. B. Gustafsson, H.-O. Kreiss, and J. Oliger, Time dependent problems and difference methods, John Wiley \& Sons, New York, 1995. MR 97c:65145

15. C. Johnson and U. Nävert, Analysis of some finite element methods for advection-diffusion problems, Mathematical and Numerical Approaches to Asymptotic Problems in Analysis (University of Nijmegen, The Netherlands, June 9-13, 1980) (L.S. Frank O. Axelsson and A. van der Sluis, eds.), North Holland Math. Stud., 47, North-Holland, Amsterdam, 1981, pp. 99-116. MR 82e:65127 
16. C. Johnson and J. Pitkäranta, An analysis of the discontinuous Galerkin method for a scalar hyperbolic equation, Math. Comp. 46 (1986), 1-26. MR 88b:65109

17. B.S. Jovanović, L.D. Iovanović, and E. Süli, Convergence of a finite difference scheme for second-order hyperbolic equations with variable coefficients, IMA Journal of Numerical Analysis 7 (1987), 39-45. MR 90a:65218

18. R.B. Lowrie, Compact higher-order numerical methods for hyperbolic conservation laws, Ph.D. thesis, University of Michigan, 1996.

19. A. Majda, J. McDonough, and S. Osher, The Fourier method for nonsmooth initial data, Math. Comp. 32 (1978), 1041-1081. MR 80a:65197

20. A. Majda and S. Osher, Propagation of error into regions of smoothness for accurate difference approximate solutions to hyperbolic equations, Comm. Pure Appl. Math. 30 (1977), 671-705. MR 57:11080

21. M.S. Mock and P.D. Lax, The computation of discontinuous solutions of linear hyperbolic equations, Comm. Pure Appl. Math. 31 (1978), 423-430. MR 57:8054

22. V. Thomée, High order local approximations to derivatives in the finite element method, Math. Comp. 31 (1977), 652-660. MR 55:11572

23. _ Negative norm estimates and superconvergence in Galerkin methods for parabolic problems, Math. Comp. 31 (1980), 93-113. MR 81a:65092

24. L.B. Wahlbin, Superconvergence in Galerkin finite element methods, Lecture Notes in Mathematics, vol. 1605, Springer Verlag, 1995. MR 98j:65083

School of Mathematics, University of Minnesota, Minneapolis, Minnesota 55455

E-mail address: cockburn@math.umn.edu

School of Mathematics, University of Minnesota, Minneapolis, Minnesota 55455

E-mail address: luskin@math.umn.edu

Division of Applied Mathematics, Brown University, Providence, Rhode Island 02912

E-mail address: shu@cfm.brown.edu

Oxford University Computing laboratory, Wolfson Building, Parks Road, Oxford OX1 3QD, United Kingdom

E-mail address: Endre.Suli@comlab.ox.ac.uk 